\title{
Impact of Beam Transport Method on Chamber and Driver Design for Heavy Ion Inertial Fusion Energy \\ D. V. Rose, D. R. Welch
}

Mission Research Corporation, Albuquerque, NM 87110

C. L. Olson

Sandia National Laboratories, Albuquerque, NM 87185

S. S. Yu, S. Neff

Lawrence Berkeley National Laboratory, Berkeley, CA 94720

W. M. Sharp

Lawrence Livermore National Laboratory, Livermore, CA 94550

and the ARIES Team

December 30, 2002 


\begin{abstract}
In heavy ion inertial fusion energy systems, intense beams of ions must be transported from the exit of the final focus magnet system through the target chamber to hit millimeter spot sizes on the target. In this paper, we examine three different modes of beam propagation: neutralized ballistic transport, assisted pinched transport, and self-pinched transport. The status of our understanding of these three modes is summarized, and the constraints imposed by beam propagation upon the chamber environment, as well as their compatibility with various chamber and target concepts, are considered. We conclude that, on the basis of our present understanding, there is a reasonable range of parameter space where beams can propagate in thick-liquid wall, wetted-wall, and dry-wall chambers.
\end{abstract}




\section{Introduction}

The U.S. heavy ion fusion (HIF) program is working towards the development of a commercially viable inertial fusion energy (IFE) power plant for generating electricity $[1,2,3]$. Intense beams of heavy ions are accelerated to several GeV energies, temporally compressed and focused onto small targets containing the fusion reaction fuel. Energy from the fusion implosion is contained within a target chamber that shields the accelerator and focusing elements from the implosion and captures a portion of the energy for conversion to electricity.

Heavy ion IFE target designs require a shaped drive pulse, that has a long duration ( $30 \mathrm{~ns})$, low intensity "foot" followed by a shorter duration ( $10 \mathrm{~ns})$, higher intensity, "main" pulse $[4,5]$. The limit to which the ion beams can be focused (the beam spot size) impacts the target gain (the ratio of the energy produced in the fusion implosion to the energy in the ion beams), and the target energy requirement, which decreases with smaller beam focal size. Consequently, estimating achievable beam spot size is an important aspect of an HIF system design. The beam spot size limits are a function of a number of parameters including the beam propagation method, chamber design, and final focus system. A number of beam propagation methods have been explored, including both ballistic and pinched propagation modes. Chamber designs are constrained in part by first-wall protection schemes, neutronics, venting, and final focus designs.

The goal of this paper is to correlate the constraints on beam propagation modes to the target and chamber designs [6, 7]. This paper is organized as follows. In Sec. 2, the first-wall chamber protection schemes are summarized along with a brief survey of the applicability of the ion beam transport modes. In Secs. 3-5, a detailed analysis of the three main beam transport modes is presented, along with parameter windows for viable chamber propagation and coupling to IFE targets. An important issue for the thick-liquid and wetted-wall concepts, recently highlighted in the ARIES studies, is the presence of aerosols in the chamber. The impact of aerosols on beam transport is assessed in Sec. 6. Conclusions are given in Sec. 7. Two special topics related to beam propagation are addressed in the appendices: Appendix A addresses the physics of a plasma plug/magnetic shutter located at the exit of the last quadrupole magnet. This device is part of the beam-line design, essential for pro- 
tection of the beam focusing system. In Appendix B, we summarize previous experimental findings related to the assisted pinched transport mode that are essential in establishing the credibility of the assisted pinched transport concept.

\section{Chamber Designs and Beam Transport Modes}

Present IFE reactor chamber designs can be broadly grouped into three classes that are characterized by the first-wall protection scheme employed; thick-liquid wall, wetted-wall, or dry-wall $[6,7,8,9]$. The thick-liquid wall chamber concept uses jetted and free-flowing molten salts such as flibe (see, for example Ref. [10]) for blast and neutron protection, as well as tritium breeding. For example, the HYLIFE-II thick-liquid wall design $[11,12,13]$ uses oscillating jets, interlaced stationary jets, and flibe vortices to protect the chamber walls and the beam entrance holes for beam and target injection $[14,15]$. The thick-liquid wall concept enables small volume chamber designs that minimize beam propagation distances within the chamber. Wetted (or thin-liquid) wall chamber designs utilize a thin liquid layer for firstwall protection. The liquid layer is resupplied after each shot. Energy flux constraints force somewhat larger chamber sizes compared with thick-liquid wall designs. The flux of photons and ions onto the liquid surfaces in both the thin and thick-liquid wall scenarios will likely result in the formation of aerosols [7]. The concentration and size of these aerosol droplets are currently under study, as well as the rate at which they can be cleared from the chamber. Some background population of aerosol droplets will likely be present in the chamber in the path of the heavy ion beams. The dry-wall designs may utilize buffer gases in the chamber to reduce the power flux but these designs have the largest chamber sizes [16]. Dry-wall designs have been integrated into several laser-driven IFE systems [17, 18, 19, 20].

The chamber design, chiefly the size and vapor pressure, determines which ion-beam transport mode is applicable. Ion-beam propagation schemes in the chamber can be broadly classed into either ballistic or pinched modes [21]. For ballistic modes, the final focus section magnetically focuses the individual beams outside of the chamber such that the beams are ballistically "aimed" at the target position. The ballistic modes, vacuum ballistic, and neutralized ballistic, have received the most study [22]. 
Vacuum ballistic transport (VBT) requires a beam perveance $K$ (ratio of space-charge to kinetic energy) that is small enough $\left(K<10^{-5}\right.$ for typical HIF parameters) to prevent the self-electric fields of the individual beams from defocusing the beams. This mode is, conceptually, the simplest, as it requires no charge neutralization and, thus, involves no plasma effects. It is also, historically, the mode of choice for most of the earlier HIF concepts. However, as economic constraints drove the ion kinetic energies lower, space-charge effects became more important. To make the space-charge forces of individual beamlets acceptable, a large number of beams, $N>500$, must be individually propagated at very low background gas densities in the chamber $(<0.1$ mtorr $)$. The individual beams overlap at the target entrance, creating a large space-charge force that may emit the collective beam spot size on target. In the present study, VBT is not assessed.

Neutralized ballistic transport (NBT) requires the presence of a plasma, either externally injected or created by ionizing the background gas. When the plasma density is significantly higher than the beam, a high degree of charge neutralization results. The number of beams can be reduced to order 100 while achieving the required beam spot. Note that VBT and NBT modes require holes in the chamber wall for each beam of radius $r_{h} \sim 5.0 \mathrm{~cm}$. Because the beam enters the chamber with a relatively large spot, the effects of gas interaction require a chamber design with low vapor pressure and short propagation distance.

Pinched mode schemes presently under consideration include assisted and self-pinched transport [23]. Assisted pinched transport (APT) uses preformed, current-carrying discharge channels to provide the pinch force that confines the individual beams. Self-pinched transport (SPT) relies on incomplete current neutralization of the beam propagating through the chamber gas to confine the beam. Both pinched modes focus many individual beams outside of the chamber to approximately the final spot size on target. The APT scheme presently under study [24] aims and focuses many individual beams into two adiabatic discharge channels outside of the chamber, one on each side. Laser-initiated discharge channels are created inside of the chamber through which two opposing high-current beams are propagated at small radii. (Additional discharge channels inside the chamber provide the necessary return current paths.) Present research into the SPT scheme is examining both multiple beam, $N \sim 100$, and few beam, $N \sim 2-10$, chamber propagation scenarios. In both pinched 
mode schemes, the beams enter the chamber through relatively small ports, $r_{h} \sim 0.5 \mathrm{~cm}$ in the chamber wall - a clear chamber design advantage. The small beam radius at the wall coupled with a strong confining force relaxes the vapor pressure and transport distance constraints.

The chamber concepts and beam transport modes presently under consideration for IFE via HIF are summarized in Table 1. The thick-liquid wall design with NBT [11] is presently the baseline U.S. HIF system design. The issue of driver and chamber interfacing has been considered extensively for the combination of NBT with thick-liquid walls [25, 26, 27], including the formulation of detailed "point designs" [28, 29]. To date, the wetted and dry wall concepts have received less attention for HIF, but recent work on the pinched transport modes suggest that HIF may also be compatible with these options. The operating windows for each of these transport modes depend on the target design.

Recent target concepts include the distributed radiator $[4,5]$, close-coupled, and hybrid [30]. The distributed radiator target is the most studied, and existing designs are robust. This design is an integral part of baseline HIF power plant scenario. The hybrid target was designed to accept large beam spot sizes, and significantly ease the requirements on the driver and final focusing. This design is well-suited to the pinched transport modes. At this point in time, the physics of the hybrid target is less well established than the distributed radiator target. The close-coupled target reduces the requirement on the driver energy, but places tighter constraints on beam spot size.

\section{$3 \quad$ Neutralized Ballistic Transport}

Serious consideration of NBT is motivated by the unacceptably large ion-beam stripping cross sections in a gas background at vapor pressure $>1$ mtorr. The enhanced line charge density of a stripped beam would lead to deleterious beam blowup. Unlike VBT, however, NBT makes use of a source of electrons to reduce the beam's effective space charge. Intensive theory and simulations have been conducted to ascertain the combined effects of beam stripping, gas ionization by the beam and photons from a hot target, as well as effects of externally injected, 
Table 1: The ARIES-IFE study of HIF. Here $R_{w}$ is the approximate reactor chamber radius, $r_{h}$ is the approximate radius of the beam entrance holes in the reactor chamber wall, $N$ is the number of ion beams, and $\epsilon$ is the beam emittance.

\begin{tabular}{|c|c|c|c|c|}
\hline \multirow{3}{*}{$\begin{array}{l}\text { Chamber } \\
\text { Concept }\end{array}$} & \multicolumn{2}{|c|}{ Ballistic Transport } & \multicolumn{2}{|c|}{ Pinched Transport } \\
\hline & \multicolumn{2}{|c|}{$\begin{array}{c}r_{h} \sim 5 \mathrm{~cm} \\
(\text { most studied) }\end{array}$} & \multicolumn{2}{|c|}{$\begin{array}{c}r_{h} \sim 0.5 \mathrm{~cm} \\
\text { (higher risk, higher payoff) }\end{array}$} \\
\hline & $\begin{array}{c}\text { VBT } \\
\text { Vacuum }\end{array}$ & $\begin{array}{c}\text { NBT } \\
\text { Plasma Generators }\end{array}$ & $\begin{array}{c}\text { APT } \\
\text { Laser } / \text { Z-Discharge }\end{array}$ & $\begin{array}{c}\text { SPT } \\
\text { Only Gas }\end{array}$ \\
\hline $\begin{array}{c}\text { Thick-Liquid } \\
\text { Wall } \\
R_{w} \sim 3 \mathrm{~m} \\
\end{array}$ & $\begin{array}{l}\text { Requires }<0.1 \text { mtorr } \\
\text { and many beams }\end{array}$ & $\begin{array}{l}\text { Baseline Approach: } \\
\text { uses preformed } \\
\text { plasma and } 1 \text { mtorr }\end{array}$ & $\begin{array}{c}1-10 \text { torr } \\
\quad N=2\end{array}$ & $\begin{array}{l}1-100 \text { mtorr } \\
N=2-100\end{array}$ \\
\hline $\begin{array}{l}\text { Wetted-Wall } \\
R_{w} \sim 4-5 \mathrm{~m}\end{array}$ & $\begin{array}{l}\text { Requires }<0.1 \text { mtorr } \\
\text { and many beams }\end{array}$ & $\begin{array}{l}\text { Possible option: } \\
\text { tighter constraints } \\
\text { on vacuum and } \epsilon\end{array}$ & $1-10$ torr & $\begin{array}{l}1-100 \text { mtorr } \\
N=2-100\end{array}$ \\
\hline $\begin{array}{l}\text { Dry-Wall } \\
R_{w} \sim 6 \mathrm{~m}\end{array}$ & Requires many beams & $\begin{array}{c}\text { Even tighter constraints } \\
\text { on vacuum and } \epsilon\end{array}$ & $\begin{array}{c}1-10 \text { torr } \\
\quad N=2\end{array}$ & $\begin{array}{l}1-100 \text { mtorr } \\
N=2-100\end{array}$ \\
\hline
\end{tabular}


plasma layers (plasma plugs). The ongoing Neutralized Transport Experiment (NTX) at Lawrence Berkeley National Laboratory (LNBL) is addressing these issues experimentally.

A simple neutralization theory developed by Olson [31] shows that, for axially available electrons such as for a beam passing through a thin plasma or a foil, an ion beam spacecharge potential can not be neutralized below a minimum potential $\phi_{\min }=m_{e} v_{i}^{2} / 2$, where $m_{e}$ is the electron mass and $v_{i}$ is the beam ion speed. This residual potential, verified in detailed particle-in-cell (PIC) simulations using LSP [32], is independent of beam spacecharge potential. The resulting neutralization fraction $f=1-\alpha m_{e} / K m_{i}$, where $\alpha$ is some constant of order unity, and $m_{i}$ is the beam ion mass. Indeed, simulations confirm that for a parallel beam $\alpha \approx 1$. For a converging beam where the neutralizing electrons are heated as they compress, simulations have calculated a slightly degraded effective neutralization with $\alpha \approx 2$ [33]. Furthermore, in the presence of a volumetric plasma with density $n_{p}>>n_{b}$, the residual potential could be further reduced by roughly the ratio $n_{b} / n_{p}$, where $n_{p}\left(n_{b}\right)$ is the plasma (beam) number density. Using an envelope solution [34] that assumes an initially linearly-focusing beam and ignores stripping and both chromatic and geometric aberrations, we can approximate the final spot size. For space-charge-dominated spot size, the final beam spot is approximated by,

$$
r_{s}=R \exp \left[\frac{-R^{2}}{2 L^{2} K^{2}(1-f)}\right],
$$

where $L$ is the beam focal length and $R$ is the beam initial radius. For a $2.5 \mathrm{kA}, 4-\mathrm{GeV}$ $\mathrm{Bi}^{+1}$ ion beam with $R=3 \mathrm{~cm}$, we can expect $r_{s}<0.1 \mathrm{~cm}$ for $f>0.92$. The Olson theory predicts $f=0.95$ for this case.

Recent work [33] has examined in detail the neutralization process of a localized plasma using 2-D PIC simulations of a "weak" beam passing through a finite length of plasma. The plasma in this regime essentially provides a space-charge-limited supply of electrons. The simulations quantify the neutralization constant $\alpha$ for a variety of conditions. The simulations model the neutralization process of a paraxial beam passing through a finite thickness plasma layer and follow the beam for an additional $25 \mathrm{~cm}$. The parameters for the $\mathrm{Pb}^{+1}$ beam are varied from 0.03 to $1.0 \mathrm{kA}$ and 1 to $9 \mathrm{GeV}$. The beam number density varies from $10^{10}$ to $3 \times 10^{11} \mathrm{~cm}^{-3}$. The 1.67 -ns duration uniform-density beam is injected through the left-hand wave-transmitting boundary at $z=0$. The plasma, extending out radially to 


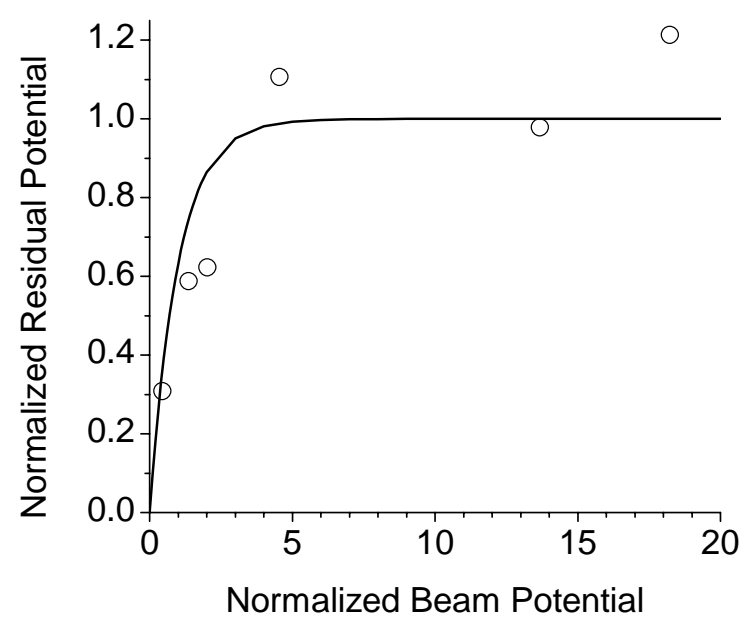

Figure 1: The residual (unneutralized) beam space-charge potential is plotted as a function of the bare beam potential. Potentials are normalized by $m_{e} v_{i}^{2} / 2$, where $v_{i}$ is the ion beam speed. Data originally presented in Ref. [33].

the outer wall and from $z=5-15 \mathrm{~cm}$, also has uniform density chosen to keep $n_{p} / n_{b}$ fixed at 10 . The initial plasma electron temperature is $5 \mathrm{eV}$. The simulation box is $3 \mathrm{~cm}$ in radius and $40-\mathrm{cm}$ long. Where the plasma is in contact with the outer wall, electron space-chargelimited emission is permitted. This boundary enables the supply of low-energy electrons to maintain quasi-neutrality of the plasma during the simulation. These PIC simulations are collisionless with no beam stripping or ionization processes.

As seen in Fig. 1, the charge neutralization for many simulations does indeed approach that of the Olson limit [31] $(\alpha=1)$. In the figure, the beam space-charge potential and the residual unneutralized beam charge are normalized by $\phi_{m i n}$. As the normalized beam potential exceeds unity, the residual charge limits to roughly unity. At small normalized beam potentials $(<1)$, the residual potential approaches the beam potential (zero neutralization limit). A fit to the simulation data, $1-\exp (-x)$ ( $x$ is the normalized beam potential), is also plotted which provides a reasonable fit to the data.

These calculations are useful in understanding the initial neutralization near the plasma. However, for a focusing beam, the problem is more complicated. As the ion beam compresses, the electron population heats degrading the neutralization somewhat as the plasma Debye 
length increases. In addition, the beam ions will ionize and be stripped to higher $Z$ by the background gas.

Two-dimensional focusing simulations have been carried out [33] to study neutralization far from the plasma. The first step is to follow a focusing driver-scale beam to target $3-6 \mathrm{~m}$ downstream of the preformed plasma. The simulations use $4-\mathrm{GeV} \mathrm{Pb}^{+1}$ ion beams with injected currents ranging from 0.25 to $4 \mathrm{kA}$ (only 1 and 4-kA currents were simulated at $6 \mathrm{~m}$ focal length). The beam perveance, used in the simulations, ranges from $10^{-5}$ to $1.6 \times 10^{-4}$ corresponding to normalized beam potentials of $4-60$. The injected beam radius is $3 \mathrm{~cm}$ for the $3-\mathrm{m}$ focal length and $6 \mathrm{~cm}$ for the $6-\mathrm{m}$ focal length. The beam normalized emittance is held fixed at $1.1 \pi$-mm-mrad. A preformed $3 \times 10^{11} \mathrm{~cm}^{-3}$ density plasma extends from 10-30 $\mathrm{cm}$ from the beam injection plane. The $13-\mathrm{cm}$ radius outer wall is permitted to emit electrons where the plasma is in contact.

Vacuum NBT transport simulations without collisions are summarized in Fig. 2. The rms radii at focus ranges from $0.6-0.8 \mathrm{~mm}$ for the $3-\mathrm{m}$ focal length, and $1-1.3 \mathrm{~mm}$ for the 6-m focal length. The Olson theory is in agreement if we use $\alpha=2$. All these spot sizes are sufficient to efficiently couple into the distributed radiator target [5]. We see that the compression and heating of the neutralizing electrons, as the beam focuses, reduces the effective neutralization from the ideal value of unity seen in the idealized calculations (Fig. 1).

In a realistic chamber environment with order mtorr vapor pressure, the ion beam interaction with the ambient gas degrades the neutralization from that of the simple theory. Recent studies of NBT have examined various sources of beam stripping as well as charge neutralization, gauging the impact of the these processes on the final spot size. Preformed plasmas at the chamber entrance have been examined as a method of improving beam space-charge neutralization as the beams enter the chamber [22, 23, 33, 35, 36, 37]. Photo-ionization of the beams and the background gas by the heated target $[38,39]$ have also been examined. Here, the deleterious process of beam photo-stripping increases the beam space charge. This is offset somewhat by photo-ionization of the background gas which creates plasma environment through which the beam can become more easily charge neutralized. 

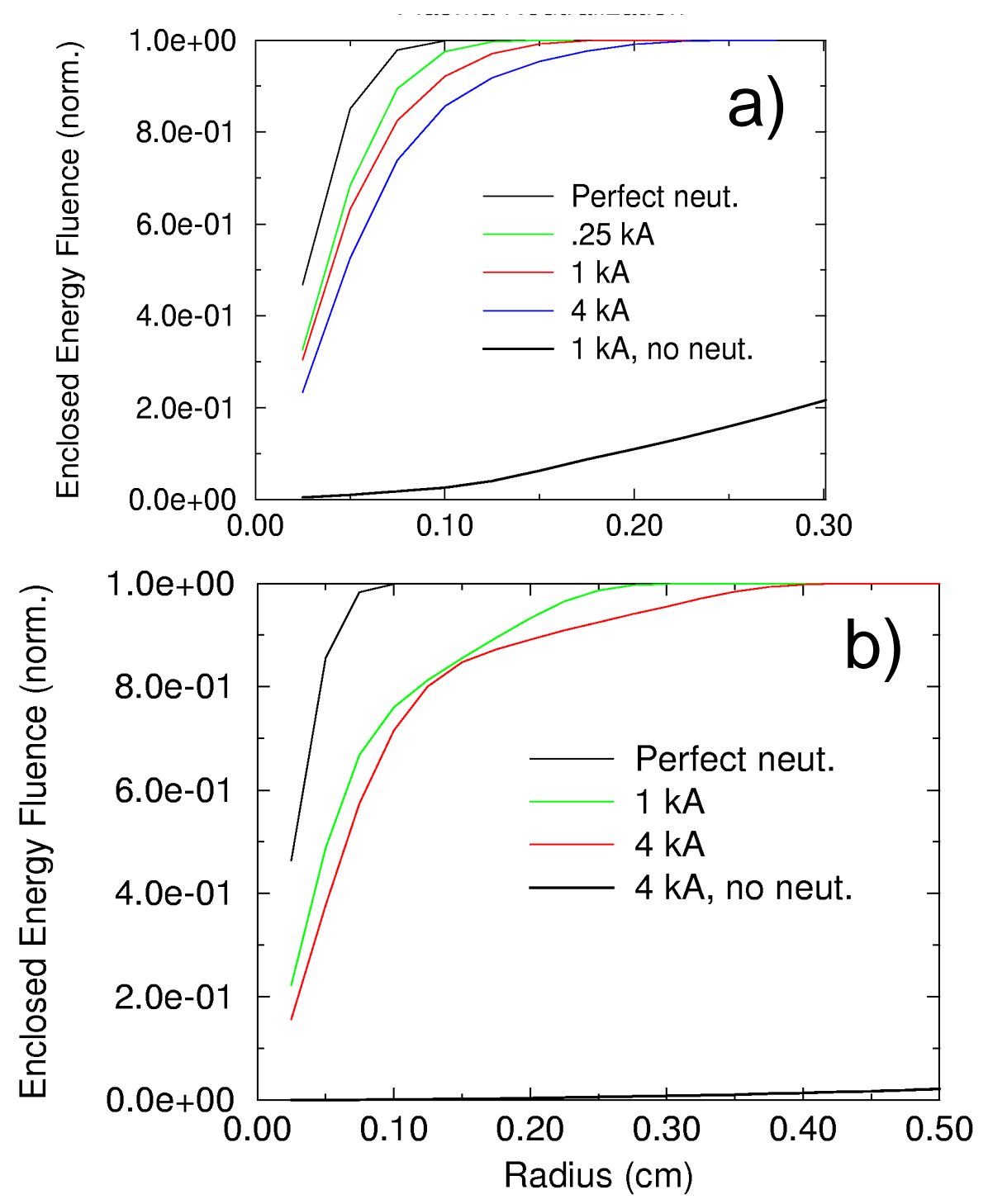

Figure 2: Time-integrated beam energy, normalized to the total injected energy, enclosed within a given radius for a) 3-m, and b) 6-m focal lengths. Data originally presented in Ref. [33]. 
An example of the impact of a pre-ionized gas on an ion beam is shown in Fig. 3. The initial plasma density is taken from separate photo-ionization calculations. The $\mathrm{Pb}^{+1}, 4^{4}$ $\mathrm{GeV}, 4-\mathrm{kA}$ beam is injected into the simulation volume with a $30-\mathrm{mm}$-mrad emittance and the background gas density is 3 mtorr of flibe. The photo-ionized plasma has a peak density of $5 \times 10^{13} \mathrm{~cm}^{-3}$ near the target and a density of $1.5 \times 10^{12} \mathrm{~cm}^{-3}$ at the chamber wall $(z=0)$. This relatively dense plasma provides good charge neutralization $\left(n_{p} / n_{b}=10\right.$ at $\left.z=0\right)$. The more highly-stripped beam ions are deflected by unneutralized space charge at beam edge [22]. The pre-ionized plasma provides $>99 \%$ neutralization at the beam focus (at $\sim 2.65 \mathrm{~m})$. However, a residual net current is observed in the simulations which results in a tight focus. The net current rises from $1 \mathrm{kA}$ after $0.84 \mathrm{~m}$ of transport to $12 \mathrm{kA}$ after $3.0 \mathrm{~m}$ of transport (after $3.0 \mathrm{~m}$ of transport the beam electrical current is roughly $20 \mathrm{kA}$ due to stripping).

The photo-ionized plasma simulation shows a dramatic improvement in the amount of beam energy delivered within $3 \mathrm{~mm}$ when compared with the neutral gas simulation, as shown in Fig. 4. These curves are also compared against a calculation of the beam profile in the absence of any electromagnetic forces on the beam (or any stripping). This is the emittance-only spot size calculation, which is only slightly higher than the photo-ionized case, illustrating the beneficial impact that the preheated target has on the main beams. Of course, photo-stripping of the beam increases the amount of beam space charge above those values obtained from only the stripping due to the background gas (see, for example, Ref. $[40])$.

Finally, multiple beam overlap at the target entrance is a concern, in part because the distributed radiator target $[4,5]$ requires that these beams cross at a common focal point away from the target face and collectively form an annular pattern on the target face. Research into the issue of multiple beam overlap for NBT is ongoing, although initial results from computational models indicate that beam spot degradation due to beam overlap is not significant.

\subsection{Operational window}

The chamber vapor pressure and radius determine the operational window for NBT. The Olson neutralization scaling suggests that the neutralization depends only on the beam 

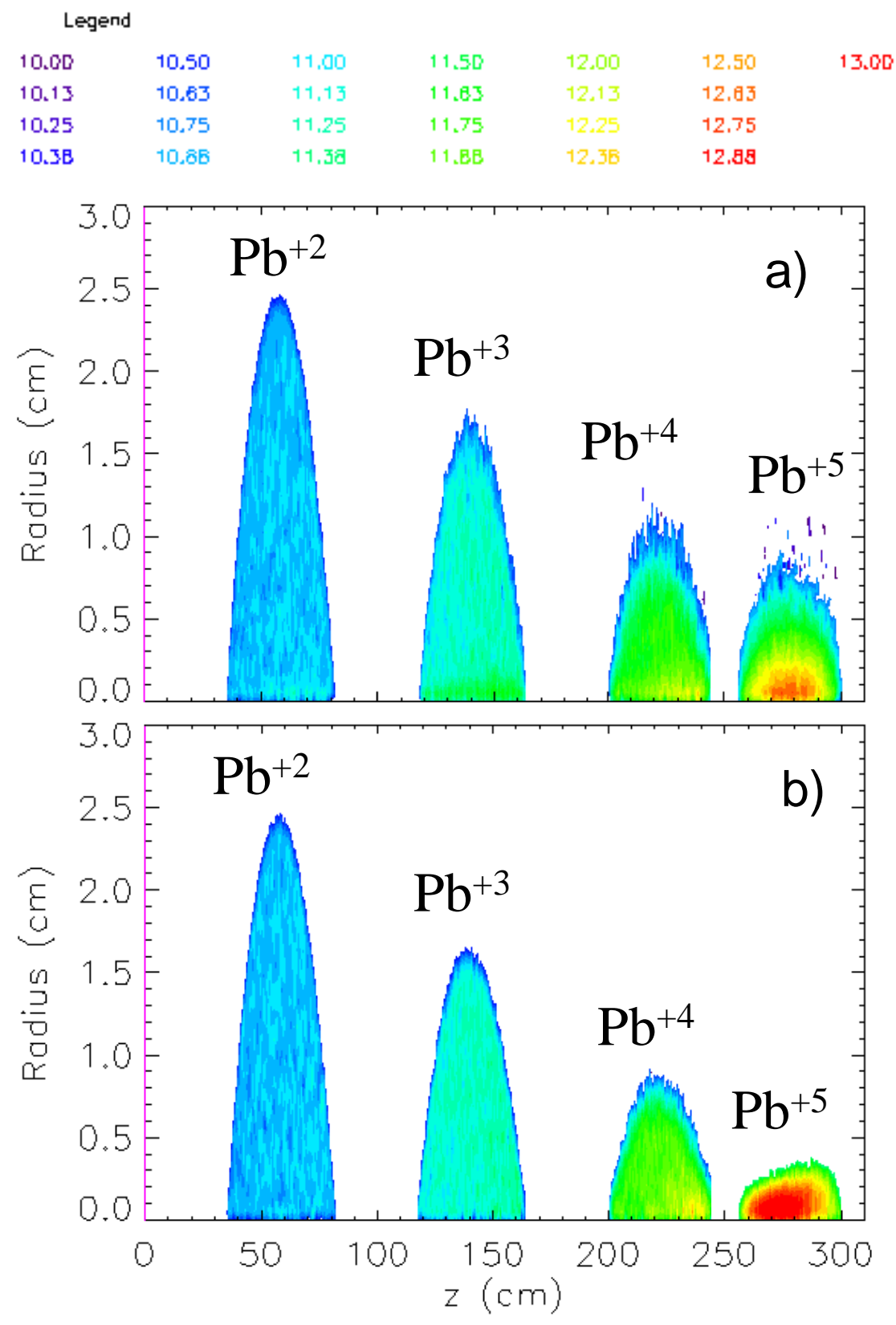

Figure 3: NBT simulation of a single 4-kA, 4- $\mathrm{GeV} \mathrm{Pb}^{+1}$ beam injected into a) background gas, and b) a partially-ionized background gas. The plasma density profile of the ionized background gas is taken from photo-ionization calculations. 


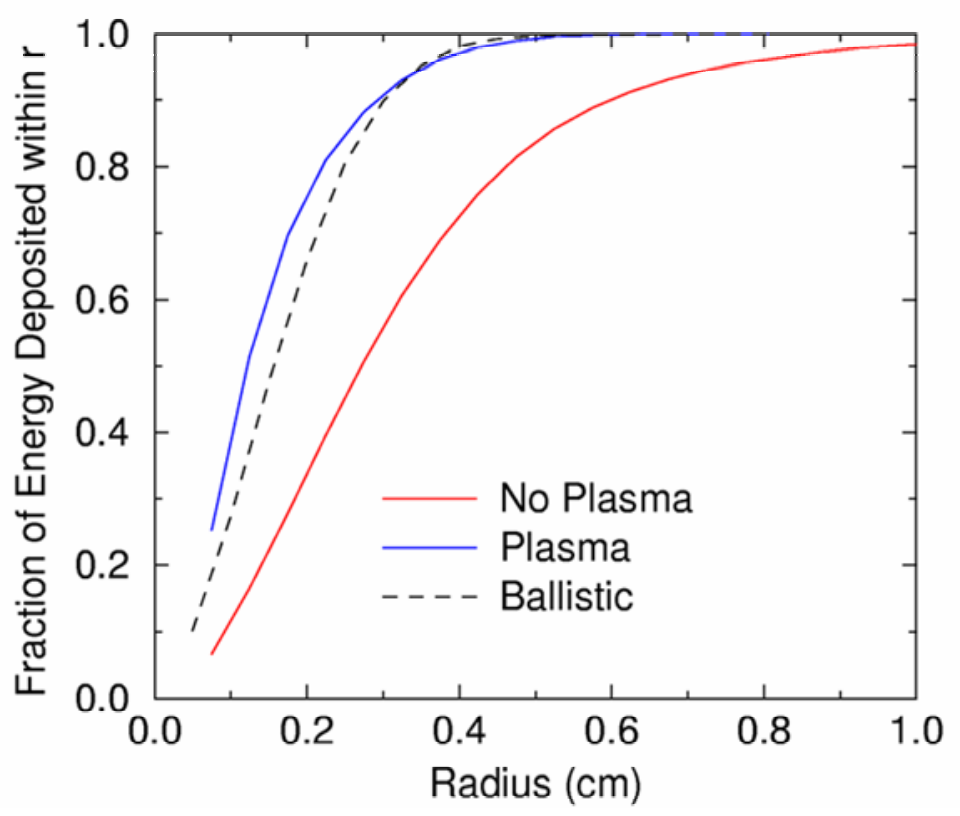

Figure 4: Comparison of the fractional beam energy deposited within a given radius as a function of radius.

velocity and is independent of beam current. Thus, for a localized supply of neutralizing electrons near the chamber entrance (preformed plasma plug) we can construct a formula for the maximum chamber radius (or beam focal length) $L_{\max }$, as a function of chamber pressure $(P)$ and the beam initial $(R)$ and final radii $\left(r_{s}\right)$. As the beam propagates through the chamber, we assume that the residual neutralization is described by the calculated $\alpha=2$. The electrons produced in a stripping event are assumed to behave as the initial entrained electrons. Also, the effects of background plasma in the chamber are neglected.

From Eq. 1 and the Olson neutralization limit,

$$
L_{\text {max }}=R \sqrt{\frac{A m_{p}}{Z_{\text {eff }} \alpha m_{e} \ln \left(R / r_{s}\right)}} .
$$

Here, $Z_{\text {eff }}=1+L_{\max } P /\left(3 L P_{\text {strip }}\right)$, is the ion charge state weighted by the distance from the target, where $P$ is the chamber flibe pressure. $L P_{\text {strip }}$ is the distance-pressure product for a single ion stripping effect (roughly 300 mtorr-cm) which we assume is constant for all ion charge states. Equation 2 can be solved for $L_{\max }$ (cubic equation) for various chamber pressures and beam $R$. As shown in Fig. 5, the chamber radius can be $300 \mathrm{~cm}$ for a 2-cm initial rms radius beam for 1-mtorr flibe. Operation with pressures as high at 10 mtorr 


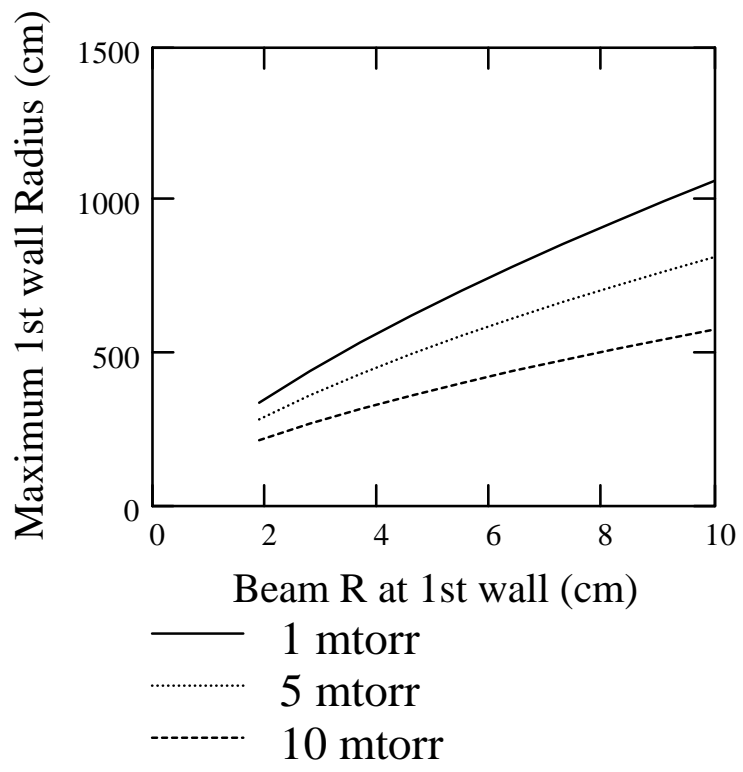

Figure 5: Operational window for NBT. The maximum allowed chamber radius is plotted as a function of initial beam radius $(R)$ for 1,5 , and 10 mtorr flibe.

requires a $>4-\mathrm{cm}$ initial beam radius for the $L_{\max }=300 \mathrm{~cm}$. Practically, the allowable size of $r_{h}$ and focusing aberrations limit the size of $R$.

The above estimate of the NBT operational window is based on the assumption that a high-density plasma $\left(n_{p}>>Z n_{b}\right)$ will not exist over the propagation length of the beam in the chamber. We do expect a large plasma density late in time (for the main pulse beams) from photo-ionization but not for the foot beams. If a high-density plasma can be produced over the entire transport distance within the chamber before the foot pulses arrive at the target, the constraint on $L_{\max }$ can be relaxed.

\section{Assisted Pinched Transport}

Formation of a stable, free-standing discharge channel is a crucial element in the design of an HIF reactor concept based of the assisted pinched mode of beam transport [24, 35, 41]. Recent experiments have demonstrated robust and stable laser-created discharge channels carrying up to $55 \mathrm{kA}$ and radii as small as $4 \mathrm{~mm}[42,43,44]$. Magnetohydrodynamic (MHD) numerical modeling of these channels is under way [44]. Efficient transport of high-current 
light ion beams in wall-stabilized discharge channels [34, 45, 46, 47] and low-current heavy ion beams in free-standing, laser-initiated discharge channels [48] has been demonstrated.

A key concept for the viability of assisted pinched transport is the introduction of a prepulse several tens of microseconds prior to the main discharge. (See Appendix B for details.) The primary function of the pre-pulse is to create a rarefied density hole. Typically, the density of the hole $\left(N_{c h}\right)$ is an order of magnitude lower than the ambient density of the chamber $\left(N_{o}\right)$. The main discharge, as well as the ion beam, is transported in this rarefied density channel. The rarefied density channel performs a number of important functions:

- It permits formation of a breakdown path along the channel rather than to the wall since the breakdown field is directly proportional to the gas density. Hence, a relatively low field along the long rarefied channel can lead to breakdown while the remainder of the chamber, with potentially much higher fields, remains insulated with the ambient gas.

- The density wall provides a mechanism for stabilization of the discharge channel against an otherwise virulent kink instability.

- Reduced density in the channel also minimizes beam energy loss since the ion stopping range is proportional to the gas density.

In Sec. 4.1, we present simulations of intense ion beams propagating in current-carrying and rarified channels. In these calculations, we fix $N_{o}$ (5 torr Xe) as well as $N_{c h}(0.5$ torr $\mathrm{Xe}$ ). Under these conditions, we show that an ion beam can couple efficiently to a hybrid target. Sensitivities of transport efficiencies to beam parameters and $z$-discharge current were explored.

In Sec. 4.2, we discuss the key effects that determine the operating window in chamber pressure and chamber size.

While the rarefied density holes make it possible to create breakdowns along the channels, in order to avoid breakdowns to the chamber walls, particularly around the beam port, we need suitably designed insulators to control the electric field distribution and minimize unwanted field enhancements. Furthermore, these special beam port insulators must be 
compatible with chamber wall protection. In Sec. 4.3, we discuss the control of breakdown in the chamber and introduce a new insulator design which may be consistent with all three chamber concepts.

\subsection{Numerical simulations of APT for HIF}

Recent analytic and numerical modeling of the APT mode [35, 41] has demonstrated a wide operational window of beam and channel parameters, consistent with previous studies $[49,50]$, that make this propagation mode feasible with all of the presently studied first-wall protection schemes. The numerical simulations have examined transport for chamber radii of $3 \mathrm{~m}$, pertaining most directly to the thick-liquid chamber design. Simulations are carried out using the hybrid PIC code IPROP [51] that includes detailed modeling of the gas breakdown for background Xe gas pressures of a few torr. These electromagnetic simulations include the effects of the beam self-fields and include a tensor conductivity model used to describe the plasma electron current. Details of the physical models used in these calculations are given in Ref. [41].

The simulation geometry includes both foot and main pulse beams, as required to couple to and drive the hybrid HIF target [30], which accepts a 5-mm beam spot size, which is larger than the distributed radiator beam spot requirement of $\sim 2 \mathrm{~mm}[4,5]$. The simulations use a 12.5-kA (particle current), 25-ns duration pulse of $3-\mathrm{GeV} \mathrm{Pb}^{+72}$ ions for one of the two foot beams and a $66.5-\mathrm{kA}$ (particle current), 8-ns duration pulse of $4.5-\mathrm{GeV} \mathrm{Pb}^{+72}$ ions for one of the two main beams. These $0.3-\mathrm{m}$ radius beams are injected into the simulation volume $10 \mathrm{~m}$ upstream of the discharge channel entrance. The foot and main beams are focused onto the discharge channel entrance and propagate ballistically across the $10-\mathrm{m}$ long drift space.

The adiabatic section of the discharge channel has an entrance radius of $0.02 \mathrm{~m}$ and tapers linearly over $1.25 \mathrm{~m}$ to the entrance of the main discharge channel that has a radius of $0.005 \mathrm{~m}$. The nominal discharge channel current is $50 \mathrm{kA}$.

Numerous simulations have been carried out to examine various sensitivities and limitations of the assisted pinched mode. The initial beam micro-divergence was increased from $1 \mathrm{mrad}$ to 1.5 and $2 \mathrm{mrad}$ to look for an upper limit for good energy transport effi- 


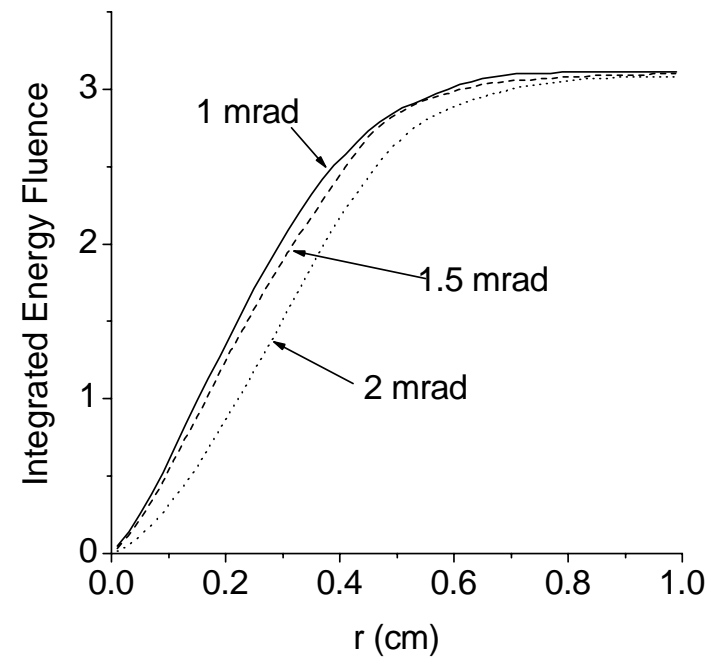

Figure 6: Integrated energy fluence on target as a function of radius for APT simulations with different initial micro-divergence values. Simulations parameters are $4.5 \mathrm{GeV} \mathrm{Pb}{ }^{+72}$, $66.5 \mathrm{kA}$ (particle current).

ciency. The results shown in Fig. 6 indicate that the transport efficiency falls slowly with beam divergence and that values less than $1.5 \mathrm{mrad}$ are adequate for these parameters (50$\mathrm{kA}$ discharge current, $4.5-\mathrm{GeV} \mathrm{Pb}^{+72}$ beam ions), which gives an upper limit on the beam micro-divergence.

The impact of discharge current on beam transport efficiency was examined using simulations with discharge current values ranging from 25 to $75 \mathrm{kA}$. As expected, the largest discharge current $(75 \mathrm{kA})$ gives the highest transport efficiency within $0.5 \mathrm{~cm}$, as shown in Fig. 7. However, the transport efficiency for the $50-\mathrm{kA}$ case is only a few percent smaller at $0.5 \mathrm{~cm}$. As the discharge current is decreased below $50 \mathrm{kA}$, a significant drop (up to 7\%) in the transport efficiency is found for these simulations parameters (1 mrad micro-divergence, $4.5 \mathrm{GeV} \mathrm{Pb}^{+72}$, and $66.5 \mathrm{kA}$ of beam particle current). Thus, a parameter window for discharge current values has been established, which is an important aspect of the reactor design.

The APT mode is also fairly insensitive to beam species, as shown in Fig. 8, which compares the $\mathrm{Pb}^{+72}$ case with a $\mathrm{Xe}^{+44}$ beam simulation. The parameters of the $\mathrm{Xe}^{+44}$ simulation are scaled to deliver the same beam energy on target (1.8-GeV foot and $2.4-\mathrm{GeV}$ 


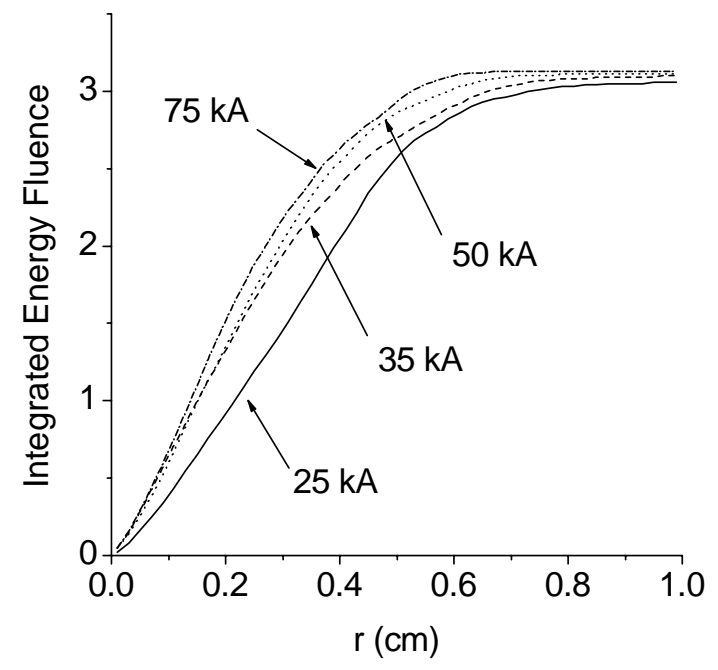

Figure 7: Integrated energy fluence on target as a function of radius for APT simulations with different discharge current values. Simulation parameters are $\mathrm{Pb}^{+72}$ foot and main pulses.

main beam, and the total electrical currents and charge-to-mass ratios are roughly equivalent to the $\mathrm{Pb}^{+72}$ simulation).

These simulations demonstrate that the assisted pinched mode is robust and that a design window exists from which a plausible reactor concept can be made.

\subsection{Control of electrical breakdown}

The mechanism for creation of the discharge channel consists of two steps. First, the electric field from the externally-applied voltage must be large enough to create breakdown along the channel, thus creating a highly conducting path. Subsequently, current flows through the conducting path. The resulting axial current provides the magnetic field for ion-beam confinement. Since the path for the discharge, together with the return current path, is relatively long ( $\sim 2$ chamber radii), one might ask why the breakdown would prefer to go along the long discharge path and not to the nearby chamber wall from the beam port (high-voltage terminal). In our design, the control of breakdown path is accomplished by a combination of a reduced density along the discharge path, and the design of an insulator around the port to move the grounded chamber wall from the high-voltage beam entry port. 


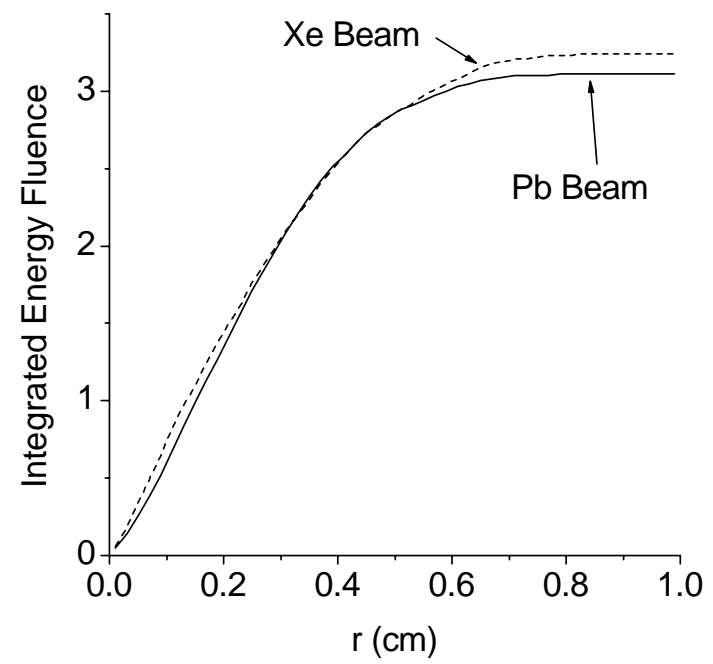

Figure 8: Integrated energy fluence on target as a function of radius for APT simulations for the $\mathrm{Pb}^{+72}$ and $\mathrm{Xe}^{+44}$ beams.

To see this, we note that the condition for breakdown to occur is

$$
E / p \geq(E / p)_{t h d},
$$

where $E$ is the electric field and $p$ is the gas density. The threshold for the electrical breakdown varies with the gas species. For Xe, breakdown occurs at $(E / p)_{t h d} \sim 60 \mathrm{~V} / \mathrm{cm} /$ torr, therefore the design goal is to manage gas breakdown by 1) creating a reduced density path in the channel path $\left(N_{c h}\right)$ such that

$$
(E / p)_{c h}>(E / p)_{t h d},
$$

and 2) maintain low values for $E / p$ everywhere else in the chamber (at gas density $N_{0}$ );

$$
(E / p)_{o}<(E / p)_{t h d} .
$$

Noting that electric field is the voltage over the path length, we can show that the desired breakdown control is attained if

$$
r_{h}>\frac{N_{c h}}{N_{0}} L,
$$

where $L$ is the discharge path length or chamber radius.

To avoid breakdown to the wall, besides making the insulator sufficiently large, we also need to shape the geometry so as to evenly distribute the electric fields throughout the 


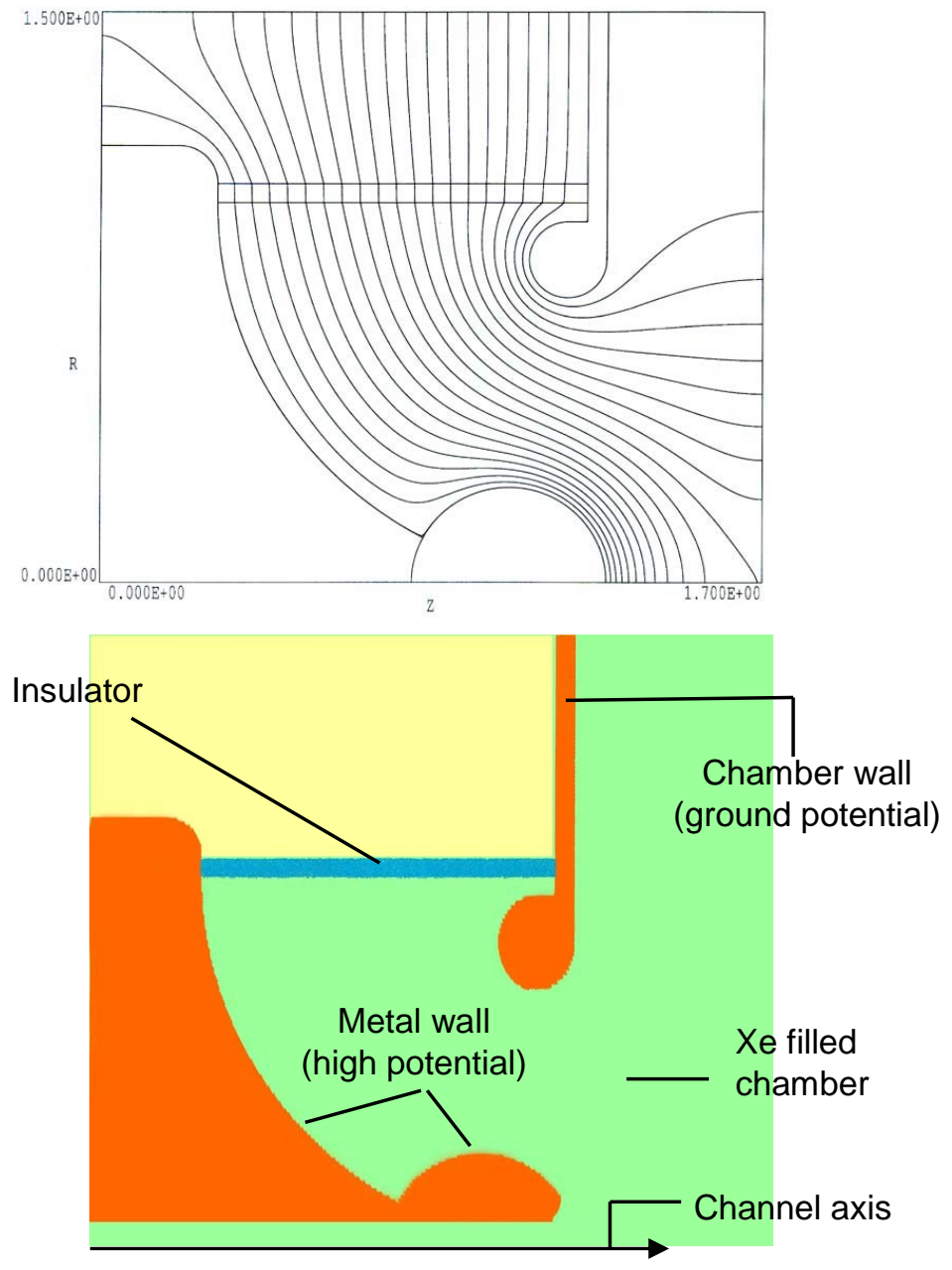

Figure 9: Beam port design schematic (bottom) and electrostatic field stress calculation (top). 


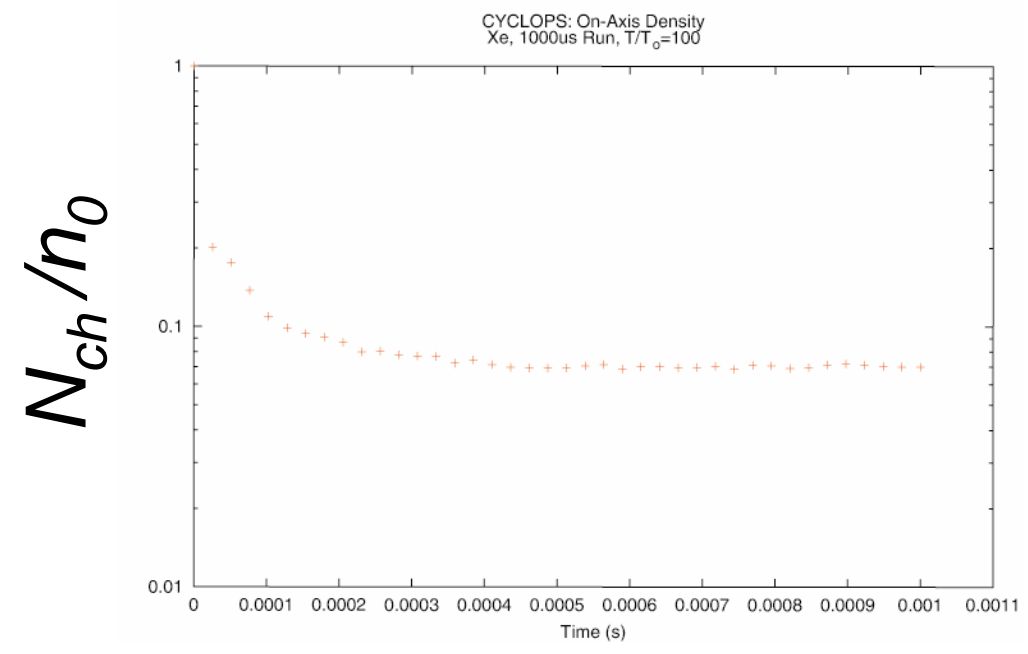

Figure 10: 1-D CYCLOPS calculation showing the ratio of $N_{c h} / N_{0}$ as a function of time.

port and insulator region. An example of an insulator port geometry designed to avoid local maxima in field enhancement is shown in Fig. 9. Earlier designs used metal sheets to provide uniform grading of the insulator. But they are not compatible with dry and thin liquid-wall chambers. The new design was constructed with compatibility with first-wall protection in mind.

The ratio $N_{c h} / N_{o}$ depends on how much energy is deposited in the prepulse. The deeper the channel, the lower the required voltage and the smaller the insulator size required to prevent breakdown to the walls. However, ultimately, processes of radiation and thermal conduction limit the achievable depth of the hole. The precise limit is still under investigation, but generally $N_{c h} / N_{o} \leq 0.1$. A calculation with a 1-D hydrodynamics code CYCLOPS is provided in Fig. 10. In this calculation, we adopt a simple model where energy is instantaneously deposited by the prepulse, and leads to a high on-axis temperature. As the initial channel temperature is raised, the final density reaches a minimum, and continues to stay at that level independent of how much energy is put into the prepulse. 


\subsection{Operational window}

The usable gas pressure range for the transport mode depends on the channel length and is

determined by two effects: the energy loss of the ion beam in the channel and the widening of the channel due to the $j \times B$ force.

Beam energy losses place a constraint on the maximum density in the channel. In general, the energy loss can be determined from

$$
\Delta E(M e V)=380\left(\frac{L}{4 m}\right)\left(\frac{N_{c h}}{0.5 \text { torr }}\right)\left(\frac{Z_{g}}{54}\right)\left(\frac{0.2}{\beta}\right)^{2}\left(\frac{Z}{64}\right)^{2},
$$

where $Z_{g}$ is the atomic number of the gas, $Z$ is the effective charge state of the stripped ion, and $\beta$ is the ion speed divided by the speed of light. Thus, for a $4-\mathrm{GeV} \mathrm{Pb}^{+x}$ ion beam traveling though $4 \mathrm{~m}$ of 0.5 torr $\mathrm{Xe}$, the ions will lose $\sim 9 \%$ of their energy.

The large current of the ion beam creates a magnetic field which acts on the return current resulting in the widening of the plasma channel. To ensure efficient beam transport, the channel expansion during the beam pulse must not be too large. As can be seen from the MHD force equation,

$$
\rho \frac{d \mathbf{v}}{d t}=-\nabla p+\mathbf{j} \times \mathbf{B},
$$

this effect increases with decreasing gas pressure. An upper bound on the channel expansion rate is obtained by neglecting the pressure term,

$$
\Delta R=\left(\frac{\mu_{0} I_{\text {beam }} I_{\text {net }}}{4 \pi^{2} R_{0}^{3} \rho}\right) t^{2} .
$$

Here $I_{\text {beam }}$ is the beam current, $I_{\text {net }}$ the discharge current, and $R_{0}$ the initial channel radius. This leads to a lower pressure limit of 0.5 torr Xe for $I_{\text {net }}=50 \mathrm{kA}, I_{\text {beam }}=3 \mathrm{MA}, R_{0}=5 \mathrm{~mm}$, $t=10 \mathrm{~ns}$, and $\Delta R \leq 1 \mathrm{~mm}$.

While Eq. 9 predicts rapid clearing of the channel at 0.1 torr or so, considerations of pressure gradient effects will make the channel expansion much more benign. The walls around the density hole will prevent the hole from enlarging under the $j \times B$ force.

Our expectation at this point is that energy loss will limit the channel density to $\sim 0.5$ to 1 torr. Given $N_{c h} / N_{0} \sim 0.1$, the ambient density in the chamber has an upper bound of $\sim 10$ torr. 
The lower bounds are more difficult to ascertain. As the pressure gets to 1 torr or so, the channel density ( $\sim 0.1$ torr) begins to fall into a regime where the simple MHD and Ohm's Law models fail. Quantitative calculations become more difficult. However, no fundamental limitation is known.

\section{Self-Pinched Transport}

The SPT concept relies on beam-driven impact ionization to initiate a plasma that provides nearly complete space-charge neutralization for the beam but incomplete current neutralization. The incomplete current neutralization must be of a magnitude such that the pinch force arising from the beam self-magnetic field is balanced with the beam kinetic pressure. Recent 2-D simulations [23] demonstrate a gas pressure window for heavy ion-beam propagation. Present work is focused on maximizing the beam energy transport efficiency for this propagation mode.

Determination of the gas pressure window is necessary for producing significant net current without the benefit of a pre-initialized channel. In Ref. [23], the conditions in which the self-pinch force of the beam is sufficient to confine the ion-beam divergence were examined for the case of Xe. The theory, described in Ref. [52], states that significant net currents are possible for an equilibrium beam profile that includes a trumpet-shaped beam head. The beam radius falls from head to body in a characteristic distance, $\tau$. The net current is optimized when $\tau$ is of order of the ionization mean-free-path $\lambda_{m f p}$. The ratio of these two quantities, $R_{t}$, is referred to as the normalized trumpet length,

$$
R_{t}=\frac{\tau}{\lambda_{m f p}}
$$

Efficiently ionizing beams have larger pinch currents at lower pressures. This optimum has also been observed in the SPT experiment on the Gamble II generator at the Naval Research Laboratory [53]. The trumpet shape is essential for providing nearly complete charge neutralization without complete current neutralization. As the beam pinches from head to body, the ionization electrons $E \times B$ drift radially into the beam leaving the positive charge of the plasma ions at larger radius. If the beam radius were constant, an ionization 
event could not provide a net neutralization without the electrons moving axially at the velocity of the beam. Thus, both charge and current neutralization would be excellent.

A pressure window in $\mathrm{Xe}$ for a $4-\mathrm{GeV}, \mathrm{Pb}^{+65}$ beam with a flat-topped radius profile and a $0.5-\mathrm{cm}$ minimum outer radius was defined in Ref. [23]. The calculations followed the beam for only $20 \mathrm{~cm}$ in order to calculate the equilibrium net current, not the beam response. The beam impact ionization cross section used is based on an analytic model [54]; $\sigma_{b}=7 \times 10^{-15} \mathrm{~cm}^{2}$. A broad propagation window in Xe was found for pressures between 10 and 150 mtorr.

We now examine in detail a beam simulation with $65-\mathrm{kA}$ current and $\tau=0.5 \mathrm{~ns}$. The beam is injected into a 1-m long, 5-cm radius tube filled with 50-mtorr Xe pressure (corresponding to $R_{t}=0.14$ ). From the above simulations we expect a $5-10 \mathrm{kA}$ net current. Such a pinch current yields a $60-80 \mathrm{~cm}$ betatron wavelength $\left(\lambda_{b}=2 \pi r_{b}\left(I_{A} / 2 I_{n e t}\right)^{1 / 2}\right)$, roughly the length of the simulation box. Earlier simulation results, presented in Ref. [23], indicated that the core of the beam pinches, but particles are lost radially early in time. The dimensionless erosion rate (length of beam lost per length propagated) reaches a value of order $10^{-3}$ between $z=75$ and $100 \mathrm{~cm}$. This value is consistent with a simple steady-state model of self-pinched beam erosion [55]. The model accounts for non-zero beam front erosion velocities and the finite energies of beam particles radially exiting from the beam. The beam in these 1-m transport simulations is not perfectly matched (particle pressure from beam divergence not balancing the pinch force) and, therefore, the beam is not in steady-state transport until $z>40 \mathrm{~cm}$, complicating the direct comparison with the model. Typically, only $61 \%$ of the beam energy is transported within a $6-\mathrm{mm}$ radius to the $1-\mathrm{m}$ position, with the bulk of the loss occurring in the first $25 \mathrm{~cm}$ of transport.

Recent simulations have examined the use of a radially tailored beam temperature profile in an effort to reduce the early time evaporative beam losses that characterized the earlier simulations presented in Ref. [23]. The temperature of the injected beam is constant from the center of the beam out to the edge of the beam where the temperature falls to zero within about one electromagnetic skin depth $\left(c / \omega_{p b}\right.$, where $\omega_{p b}$ is the beam plasma frequency). A sample simulation result is shown in Fig. 11 which shows a strong reduction in the number of ions that are lost radially. Figure 12 shows the beam rms radius from this same simulation 


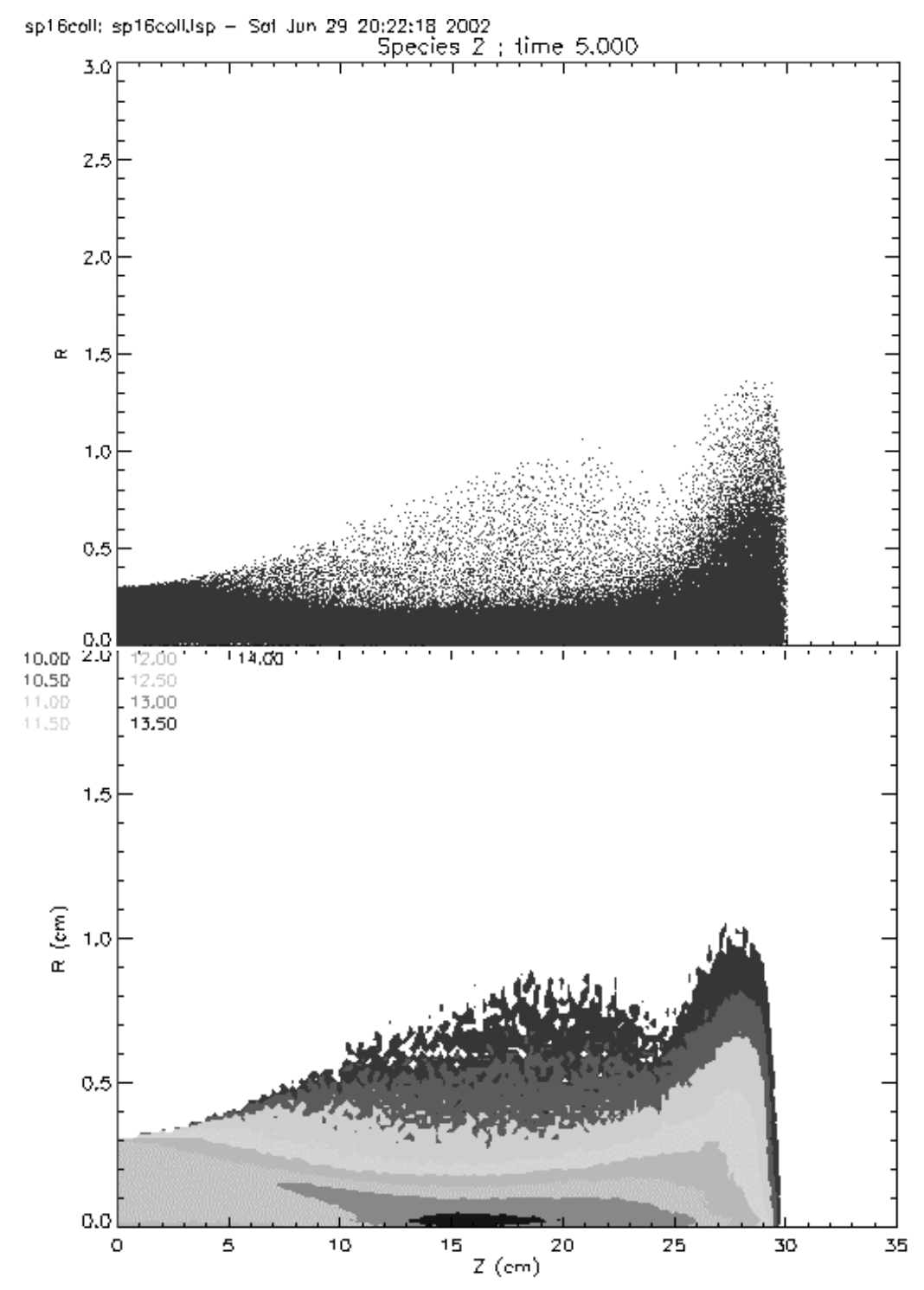

Figure 11: a) Particle positions at $5 \mathrm{~ns}$, and b) contours of beam number density at the same time from an SPT simulation. 


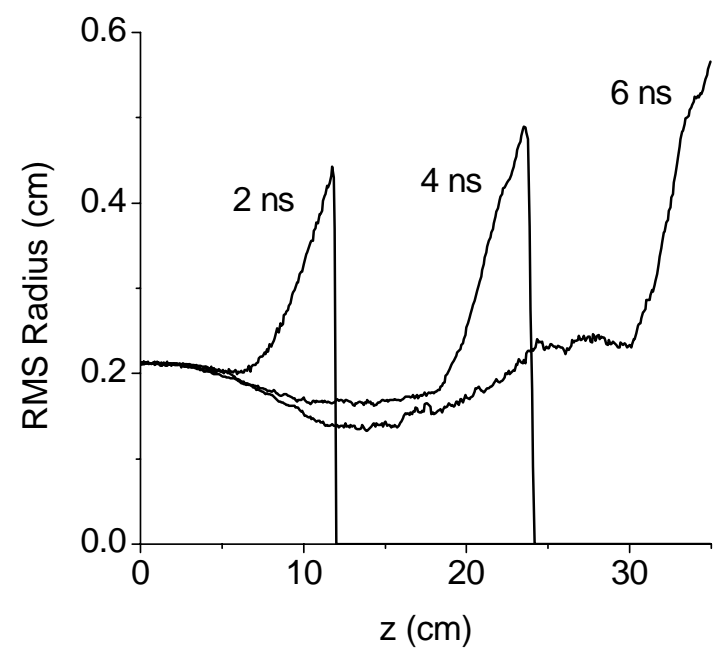

Figure 12: Comparison of rms radii at different times from an SPT simulation.

at three times. These results indicate pinched transport, but further work is required to find an optimal matching condition.

SPT is still the most speculative of the three transport modes. A broad pressure window exists, but optimization of this transport is still being studied. The LSP simulations are encouraging in that a stable pinched 2-D equilibrium is found. The calculated total transport efficiency is not acceptable due to early transient losses. If this initial loss can be avoided, the calculated late-time erosion rate would yield adequate energy transport efficiency. This needs to be explored in more detail. The 1-m-long 2-D simulations already require several million particles and 100,000 cells. Transporting the beam over the required 3-m distance in simulation will be challenging. Important questions not addressed to date include 3-D stability, aiming, and tracking.

\section{Impact of Aerosols on Beam Transport}

One potential effect that has been identified with the wetted and thick-liquid wall protection schemes is the formation of aerosols after the initial target explosion [7]. The likelihood of the formation of micron-scale-size liquid density droplets leads to an assumption that some aerosol density will be present in the chamber for subsequent shots. Therefore, estimates 
of the impact of aerosols on beam propagation in the chamber must be made. Aerosols can strip and scatter the ion beams. They can be ionized by the beam through impact or breakdown in the self-fields of the beam. They can also become charged and behave as a plasma themselves. In this section, a simple model is presented along with PIC simulations of the impact of the aerosols on beam propagation.

\subsection{Equivalent Density Model for Aerosols}

We begin with the assumptions that aerosol droplets are identical liquid spheres of radius $r$, and that they can be packed as cubes so that $(2 r)^{3}=f / n_{\text {aero }}$, where $f$ is the volume-fill fraction and $n_{\text {aero }}$ is the aerosol number density. This can be rewritten as $n_{\text {aero }} r^{3}=f / 8$. Thus for an aerosol density of $10^{9} \mathrm{~cm}^{-3}$, droplet sizes can range from 0.5 to $5 \mu \mathrm{m}$ for $f$ between 0.001 and 1. For beam interaction effects on aerosols that only depend on line-integrated density (such as mass stopping power, small-angle scattering, and stripping), an equivalent density can be defined; $n_{e q}=n_{\text {aero }} N_{d}$, where $N_{d}$ is the number of atoms per droplet or $N_{d}=(4 / 3) \pi r^{3} n_{l}$, where $n_{l}$ is the liquid number density. Therefore, $n_{\text {aero }} r^{3}=3 n_{e q} / 4 \pi n_{l}$. From this last expression, $n_{e q}$ can be calculated for mass stopping power and scattering.

The classical, nonrelativistic mass stopping power is [56]

$$
-\frac{d E}{d x}=\frac{Z^{2} e^{4} n Z_{g}}{4 \pi \epsilon_{o}^{2} \beta^{2} c^{2} m_{e}} \ln \left(\frac{b_{\max }}{b_{\min }}\right),
$$

where $n$ is the droplet neutral number density, and $b_{\max }\left(b_{\min }\right)$ is the maximum (minimum) collision parameter. The allowable energy loss limit can be expressed as $(-d E / d x) L=\eta E_{o}$, where $L$ is the beam propagation distance (here the chamber radius), $\eta$ is the fractional energy loss allowable, and $E_{o}$ is the beam kinetic energy as it enters the chamber. Therefore, the equivalent, allowable aerosol density is

$$
n_{e q}=\frac{m_{e} \beta^{2} c^{2} \eta E_{o}}{4 \pi \epsilon_{o}^{2} Z^{2} e^{4} Z_{g} L}\left[\ln \left(\frac{b_{\max }}{b_{\min }}\right)\right]^{-1} .
$$

For the sample case of $4-\mathrm{GeV} \mathrm{Pb}{ }^{+1}, L=5 \mathrm{~m}, \eta=0.1, Z_{g}=3$, and $\ln \left(b_{\max } / b_{\min }\right)=5$,

$n_{e q}=4.2 \times 10^{21} \mathrm{~cm}^{-3}$, which suggests that mass stopping due to aerosols is not likely to be a factor for ballistic neutralized transport. 
Multiple small-angle scattering [57] of the beam arising from the presence of aerosols can also be effectively treated as a function of line-integrated density. The rms scattering angle is $\Theta_{r m s}=\sqrt{\left\langle\Theta^{2}\right\rangle}$ and

$$
\left\langle\Theta^{2}\right\rangle=2 \pi n L\left(\frac{2 Z Z_{g} e^{2}}{M \beta^{2} c^{2}}\right)^{2} \ln \left(\frac{b_{\max }}{b_{\min }}\right),
$$

where $M$ is the beam ion mass. The allowable limit on multiple small-angle scattering can be expressed as $\Theta_{r m s}=\eta r_{s} / L$, where $r_{s}$ is the required beam spot size. This expression can be rewritten to obtain the equivalent, allowable number density

$$
n_{e q}=\left(\frac{\eta r_{s} M \beta^{2} c^{2}}{2 L Z Z_{g} e^{2}}\right)^{2}\left[2 \pi L \ln \left(\frac{b_{\max }}{b_{\min }}\right)\right]^{-1} .
$$

For the same sample parameters used above and with $r_{s}=0.2 \mathrm{~cm}, n_{e q}=1.1 \times 10^{18} \mathrm{~cm}^{-3}$, which also suggests that multiple small-angle scattering due to aerosols is not likely to be a factor for ballistic neutralized transport.

These limits on the effective number density $n_{e q}$ are plotted in Fig. 13 as a function of $Z$. The curves help to define design windows for beam and chamber interfaces. NBT is primarily limited by stripping of the beam and an upper limit of $n_{e q} \sim 4 \times 10^{13} \mathrm{~cm}^{-3}$ is used. This approximate value has been determined from PIC simulations of heavy ion beams propagating in background gases over 3-m distances [22, 23, 35, 36, 33, 37]. The SPT window assumes an upper limit of approximately 100 mtorr as determined from PIC simulations (see Sec. 5). An average beam charge state of $\sim 65$ is assumed. The APT window assumes a higher average charge state, in part because the discharge channel operates in higher gas densities ( $\sim 1$ torr, see Sec. 4$)$.

Estimates from the literature on the initial net charging of aerosol droplets $[58,59,60]$ (prior to interactions with charged particle beams) suggest that droplets may be either positively or negatively charged and have small net charge-to-mass ratios. Thus, net potentials from the initial aerosol population are likely to be very small, and not a concern for beam neutralization.

For NBT, the dominant limit on aerosol density, as with the uniform gas pressure, is driven by beam ion stripping. This limit is simply calculated from the equivalent integratedline gas density with the same constraints as shown in Fig. 5. Given sufficient plasma neutralization of the beam entering the chamber, adequate beam focusing for NBT can 


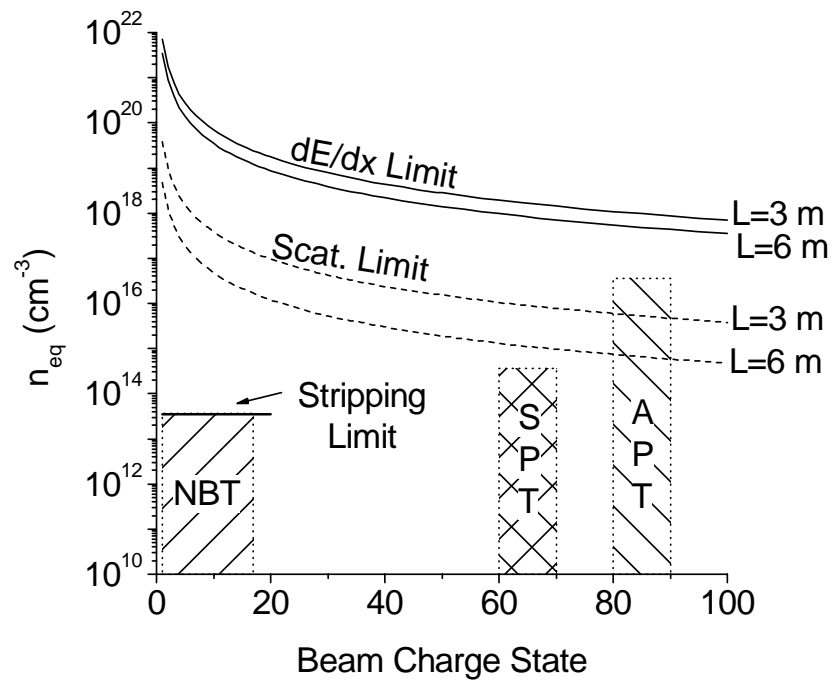

Figure 13: Sample limits $n_{e q}$ as a function of the ion-beam charge state. The parameters for these calculations are $\eta=0.2, r_{s}=0.002 \mathrm{~m}, E_{o}=4 \mathrm{GeV}, Z=3$.

be achieved for an acceptable range of flibe line-integrated pressure. The calculation of the equivalent aerosol density limits suggest that the APT mode should only be affected by additional beam scattering from the aerosol. The smallest equivalent aerosol density (assuming $\theta \simeq 0.1$ ) then lies between $100 \mathrm{mtorr}$ for $6 \mathrm{~m}$ of transport to 1 torr for $3 \mathrm{~m}$ of transport. SPT may also have a further limit on aerosol density that comes from the background gas pressure only. Thus, the equivalent aerosol pressure must be less than the background gas pressure for optimal transport, which lies approximately between 10 and 100 mtorr.

\subsection{Single Droplet Simulations}

PIC simulations using LSP [32] were carried out to assess the effects of aerosol droplets on HIF beam propagation, in particular the net charge of the droplet and the beam interaction. The simulations were designed to examine the time-dependent evolution of a single micronscale-size cylindrical droplet impacted by a uniform current density heavy ion beam. The LSP simulations include physics-based models for impact ionization and beam ion stripping. These ion processes along with co-moving electrons can remove or deposit charge onto the droplet. The simulation geometry is $2-\mathrm{D}$, axisymmetric $(r, z), 200-\mu \mathrm{m}$ long with a $100-\mu \mathrm{m}$ 


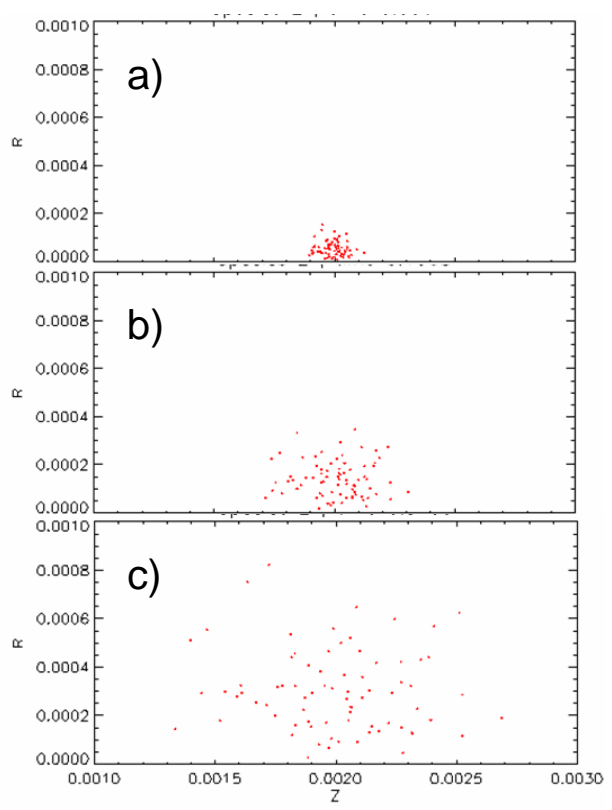

Figure 14: Neutral particle positions at a) 67 ps, b) 167 ps, and c) 375 ps from the $0.2-\mu \mathrm{m}$ droplet simulation.

radius. These electrostatic simulations use conducting boundary conditions enclosing an evacuated chamber (except for the droplet). The cylindrical droplet is initialized on-axis at the center of the simulation and is composed of $10^{21} \mathrm{~cm}^{-3}$ neutral $\mathrm{H}_{2}$ molecules at $1 \mathrm{eV}$. Droplet sizes of 0.2 to $5.0-\mu \mathrm{m}$ radius were simulated. A $3.8-\mathrm{GeV} \mathrm{Pb}^{+1}$ ion beam is injected longitudinally with a current density of $320 \mathrm{~A} / \mathrm{cm}^{2}$. A co-moving electron beam with the same velocity and number density is co-injected with the ion beam.

On time scales of less than $1 \mathrm{~ns}$, the simulations show that the ion beam heats and blows apart the droplet. The neutrals are heated to $\sim 1.2 \mathrm{eV}$, and the droplet expands at roughly $2.0 \mathrm{~cm} / \mu \mathrm{s}$, as shown in Fig. 14. For this simulation, the initial droplet size is $0.2 \mu \mathrm{m}$. The plasma formed at the droplet site expands due to ambipolar diffusion from 6-eV electrons created in ionization events (initial electron energies are of order of the neutral ionization potential). The plasma ion density at three times is shown in Fig. 15. The peak electrostatic potentials remain at $\sim 5 \mathrm{eV}$, but the extent of the potential well surrounding the droplet grows with the expanding $10^{15}-10^{16} \mathrm{~cm}^{-3}$ density plasma. After the initial dismemberment and ionization of the droplet, subsequent beam ions are weakly affected by this potential well. These results suggest that there should be no measurable electrostatic effect on the 


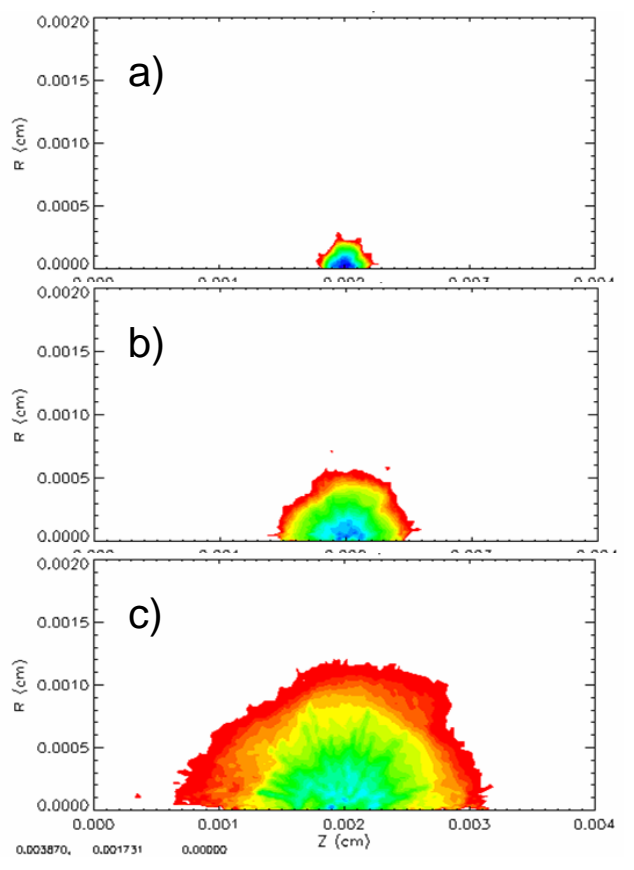

Figure 15: Plasma ion particle densities at a) $67 \mathrm{ps}$, b) $167 \mathrm{ps}$, and c) 375 ps from the $0.2-\mu \mathrm{m}$ droplet simulation.

beam. Also, while beam ion stripping can be large for a few beam ions, stripping events in subsequent ions are quickly reduced as the droplet expands, and the stripping effect approaches the limit of continuous line-gas density.

The simulations for larger droplets (up to $5 \mu \mathrm{m}$ ) give results similar to the $0.2-\mu \mathrm{m}$ case presented above. These results suggest that in regimes of interest for HIF the effect of droplets can be calculated purely from their line-integrated density. In all cases, droplet charging effects were observed to be very weak, with peak potentials between 5 and $8 \mathrm{eV}$. Small vaporized droplets (with scale-sizes less than $0.1 \mu \mathrm{m}$ ) will expand to near uniform density on beam time scales $(\sim 10 \mathrm{~ns})$ for $>10^{6}$ droplets uniformly spaced. From these simulations, we conclude that for NBT, no further constraints on aerosol properties beyond that of line-integrated density for aerosols are found.

Another possible concern is the possible micro-breakdown of a fine aerosol mist and the impact that this might have on the net beam neutralization. Given that the droplets in the path of the ion beams quickly form plasmas, droplets outside of the beam paths could then be subject to the net fields of the beams. For NBT, the individual beams must be 
well-neutralized so that the net electrostatic potential and radial electric fields at the beam edge are small relative to the bare beam values. The minimum net potential is roughly $\sim 10 \mathrm{keV}$ for a $4-\mathrm{GeV}, \mathrm{Pb}^{+1}$ ion beam [31]. If the aerosol mist outside of the beam were to break down, electrons drawn radially into the beam from the mist would not reduce the net potential substantially, in the limit that this mist has a roughly symmetric density around each beam. Therefore, the possibility that a micro-breakdown of a fine aerosol mist disrupting the neutralization of an ion beam appears to be negligible.

Finally, the impact of plasma-like effects arising from charged aerosol particles is examined. Treating the charged droplets as a separate plasma species, the charge-to-mass ratio is very small (assuming charge states of $\sim \pm 1$ ), so that the plasma frequency for this species is very small. Debye shielding can occur (e.g., outside of the beam envelope) but with little effect on the beam. Plasma waves and instabilities can also occur, but the time scale for relevant growth rates is very long compared with the beam pulse duration (see, for example, Ref. [61]). Thus, the impact of plasma-like effects of charged aerosol droplets on HIF beam transport appears to be negligible.

\section{Conclusions}

The successful design of an HIF power plant requires careful integration of the target design, beam transport mode, and chamber design. In this paper, the three main beam transport modes and first-wall chamber designs have been considered. The analysis suggests several combinations of first-wall chamber protection schemes and beam transport modes are viable. The transport mode analysis has included constraints associated with relevant target designs. The combination of NBT and thick-liquid wall protection remains the baseline HIF scheme. The analysis is also consistent with recent beam-line designs which include magnet shielding and debris mitigation associated with the large ports in the chamber walls. Also, the present analysis suggests that NBT may be consistent with dry and thin-wetted wall chamber designs as well.

New beam port designs discussed here have suggested that APT, along with the hybrid target, is compatible with all three first-wall protection schemes. APT reduces many final 
focus/chamber integration complexities by having fewer and smaller beam entrance holes in the chamber. Analysis of this transport mode and chamber interfacing suggests significant flexibility, with reasonably-large design window openings.

Recent SPT modeling suggests a wide chamber pressure window making this transport mode consistent with all of the first-wall chamber protection schemes. Since this transport mode does not require the additional complexity of establishing a discharge channel for the beam to propagate in, SPT has the simplest chamber interface of the three transport modes considered here. However, the physics of this propagation mode is complex, making accurate estimations about beam focal size limits and energy transport efficiency more difficult. Research into this transport mode is continuing.

\section{A Beam Line Design for NBT}

NBT transport requires many large area beam entrance holes in the target chamber. This important chamber interface issue requires careful beam-line designs outside of the chamber that 1) allow the ion beam to enter the chamber, 2) provide electron neutralization of the beam space charge, 3) inhibit rapid upstream motion of electrons, and 4) provide protection from chamber plasmas and gas that are expanding outward after the fusion explosion. A new concept for the beam-line design $[62,63]$ has been developed that addresses these issues with two key components: a dipole magnet just downstream of the final focus magnets and a plasma with a time-ramping density.

We have identified two technical issues relating to the particle motion up the beam line. First, as the ion beam approaches the plasma plug (which provides electron neutralization to the beam), a nonuniform density of plasma electrons with current approaching that of the ion beam is pulled upstream. The anharmonic electrostatic potential of the electrons drives the beam to unacceptably high emittance for beam currents above $2 \mathrm{kA}$. Using LSP, we have examined the emittance growth from a $6-\mathrm{cm} 4-\mathrm{GeV} \mathrm{Pb}^{+1}$ beam entering a plasma region. The plasma was modeled as a zero potential surface with space-charge-limited emission of electrons. Shown in Fig. 16, the upstream electron current and the resulting ion emittance

increase faster than linear with the ion-beam current from $250 \mathrm{~A}$ to $4 \mathrm{kA}$. At $4 \mathrm{kA}$, the 


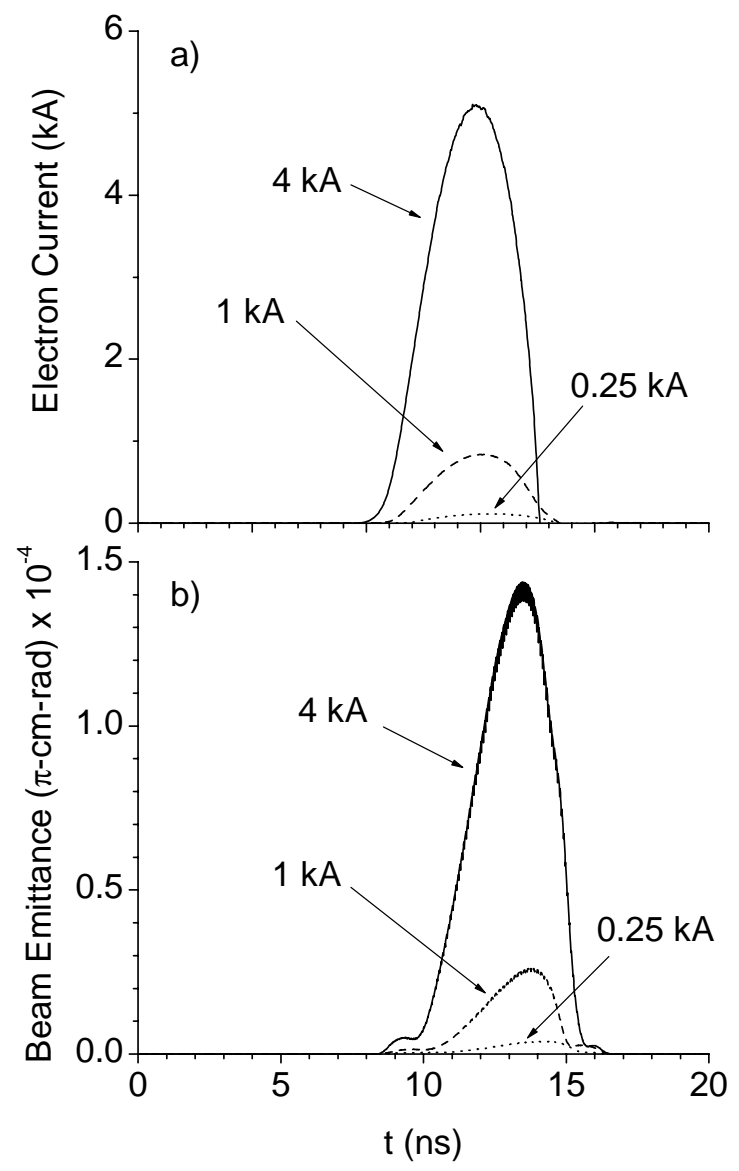

Figure 16: The a) electron current, and b) ion beam emittance entering the plasma are plotted versus time for a $6-\mathrm{cm} 1-\mathrm{GeV} \mathrm{Pb}^{+} 1$ beam in an 8 -cm radius beam line. LSP simulations include ion beam currents of $0.25,1$, and $4 \mathrm{kA}$.

emittance growth is unacceptable, exceeding $1 \pi$-mm-mrad. Fortunately, we have found in simulations that a $2-\mathrm{kG}$ dipole magnet of 5 -cm axial extent and a plasma with density ramping from $10^{12}$ to $10^{15} \mathrm{~cm}^{-3}$ in $1.0 \mu$ s are sufficient. Also, recent estimates $[62,63]$ suggest mass fluxes $6 \mathrm{~m}$ from the target that are not sufficiently low to prevent significant debris contamination on the beam-line tube metal surfaces in the final-focus magnet region. Our approach is to use a magnetic dipole to suppress upstream electron motion and divert the debris into a liquid vortex. As long as the magnetic field required is sufficiently low not to steer the heavy ion beam, this dipole may provide a simple method to mitigate both problems. 
The dipole magnetic section must provide a dual purpose. The field slows the upstream motion of the plasma electrons in 3-D LSP simulations. We have found that a 2-kG dipole over a 5-cm length is sufficient to inhibit upstream electron current. Shown in Fig. 17, the electron motion is increasingly suppressed as the dipole field is increased from 1 to $4 \mathrm{kG}$. At $4 \mathrm{kG}$, the electrons are completely confined with little growth in emittance. The emittance does increase somewhat for $1 \mathrm{kG}(0.15 \pi$-mm-mrad) and $2 \mathrm{kG}(0.05 \pi$-mm-mrad), but not enough to significantly affect the beam spot size on target.

The effectiveness of the magnetic dipole as a debris sweeper has also been studied using 3-D LSP simulations. The gas density and temperature profiles are highly time-dependent. Recent TSUNAMI calculations $[62,63]$ predict the following peak values: a molecular density of $1.7 \times 10^{15} \mathrm{~cm}^{-3}$, a temperature of $1.5 \times 10^{5} \mathrm{~K}(13 \mathrm{eV})$, an axial speed of $64 \mu \mathrm{s}^{-1}$, and a radial speed of $4.2 \times 10^{2} \mu \mathrm{s}^{-1}$. Preliminary LSP simulations have been carried out to examine the effectiveness of the magnetic shutter at stopping a drifting plasma front. The simulations use higher temperature and drift velocity plasmas than predicted by recent calculations for computational efficiency.

A thermal, drifting plasma is initialized inside a $30-\mathrm{cm}$ long, cylindrical metal pipe, $5.0 \mathrm{~cm}$ in diameter. This is roughly one-half the diameter of the beam-line tube design. The 100-eV hydrogen plasma has an initial density of $10^{14} \mathrm{~cm}^{-3}$ and a length of $10 \mathrm{~cm}$. The plasma is initialized with a drift speed of $9 \mathrm{~cm} / \mu \mathrm{s}$. The $1-\mathrm{kG}$ magnetic field is perpendicular to the cylinder axis and occupies the second-half of the pipe length. Figure 18 displays contours of the plasma density in the plane $(x=0)$ at the center of the pipe. Figure 18a) shows the initial location of the plasma. After $250 \mathrm{~ns}$, the effectiveness of the magnetic field in impeding the progress of the drifting plasma can be seen [Fig. 18b)].

The slowing down of the plasma front can also be seen more quantitatively in Fig. 19b), which plots the plasma density along the center of the pipe at different times. This can be contrasted with the results of a simulation without an applied magnetic field, Fig. 19a). The simulation also shows, Fig. 18b), the detachment of a small, localized clump of plasma that penetrates $\approx 8 \mathrm{~cm}$ or so into the magnetic field region. The penetration of low- $\beta$ plasma, where $\beta$ is the ratio of the kinetic-to-magnetic pressure, across a magnetized vacuum is an area of extensive research. Comparison with many plasma penetration models, such as 

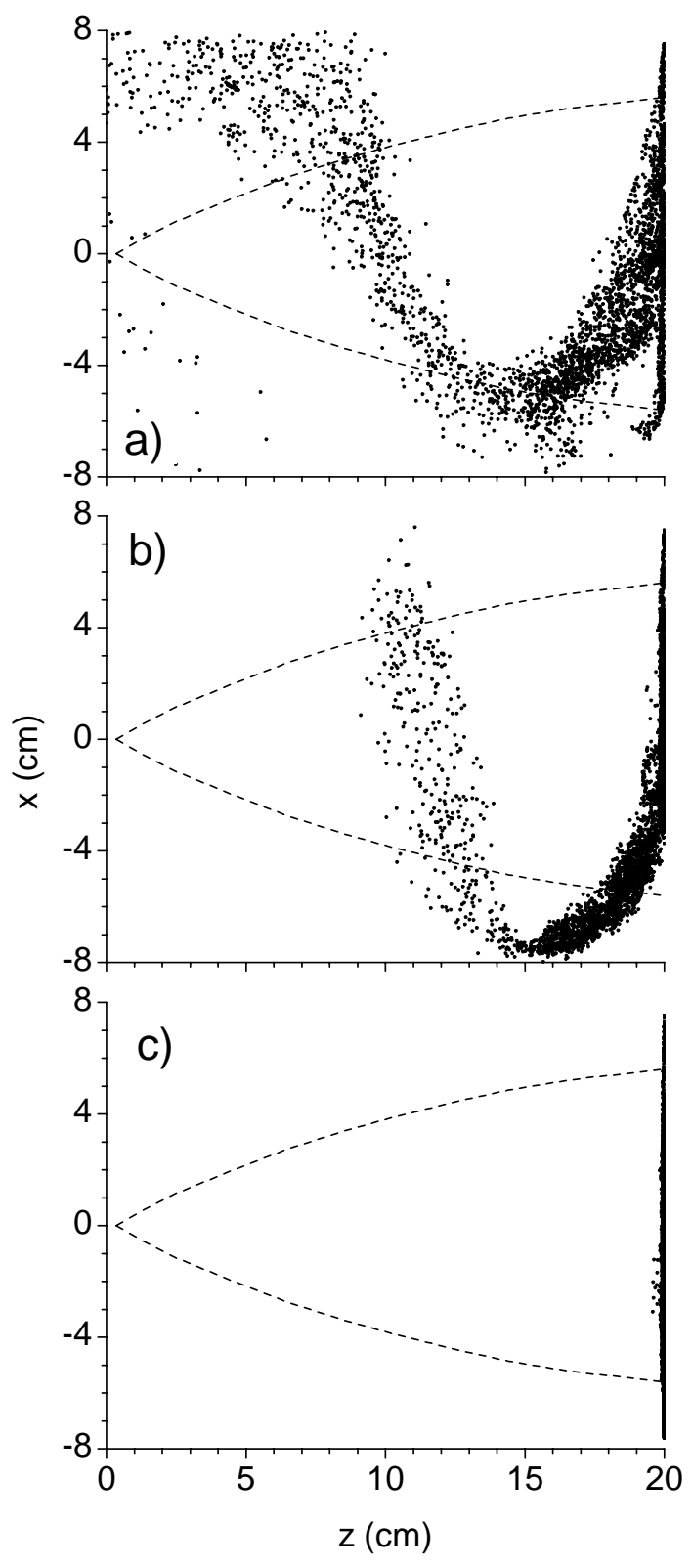

Figure 17: Snapshot of the plasma electrons upstream of the plasma plug are shown after the peak beam current has entered the plasma. The dipole field extends from $15-20 \mathrm{~cm}$ and has a) $1-k G, b) 2-k G$, and c) 4-kG peak. The dashed line represents the ion-beam profile. 


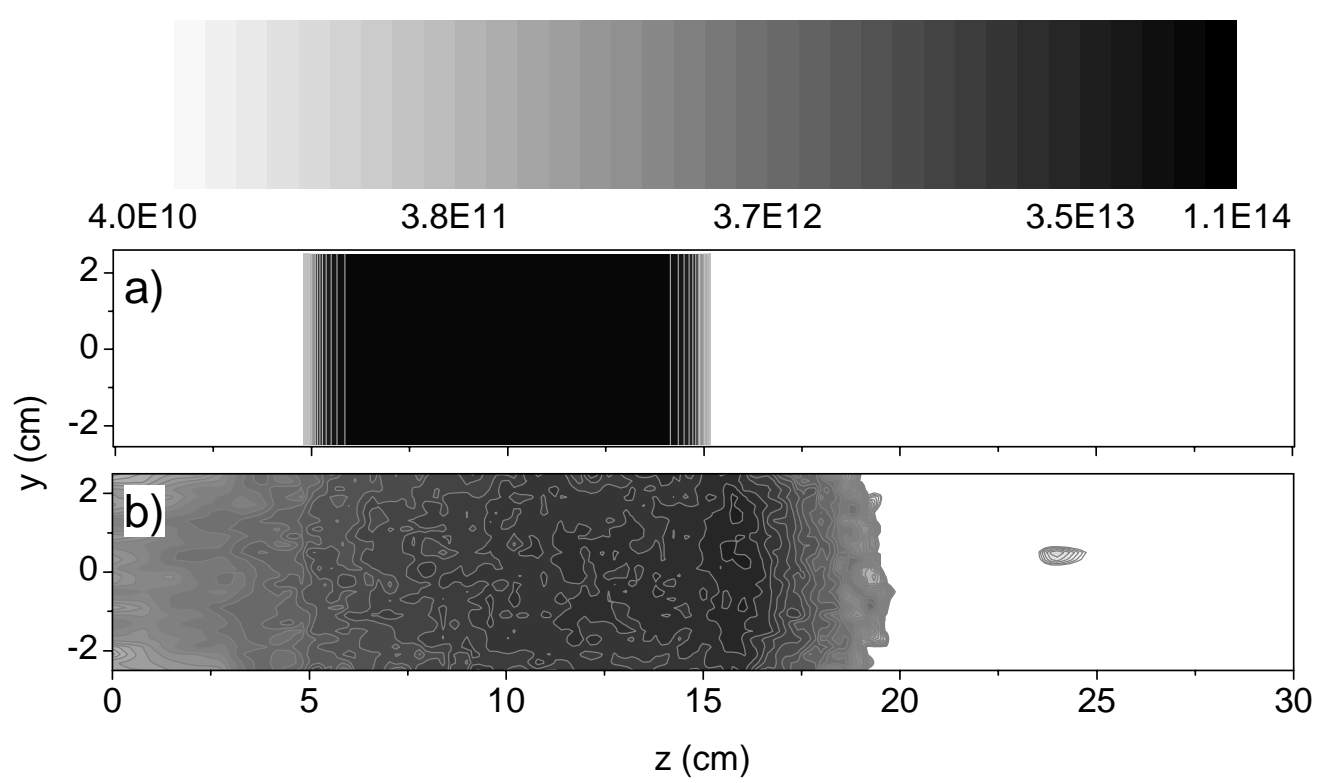

Figure 18: a) Initial plasma ion number density contours $\left(\mathrm{cm}^{-3}\right)$ for $x=0$ from the LSP simulation, and b) after $250 \mathrm{~ns}$. The applied magnetic field region extends from $z=15 \mathrm{~cm}$ to $z=30 \mathrm{~cm}$.

described in Ref. [64], requires higher fidelity simulations, which are presently being carried out.

\section{B A Review of APT Experimental Efforts}

\section{B.1 Experimental setup}

APT was first studied as a final transport mode for light ion-beam fusion (see, for example, [47] and references therein). The concept was then transferred to the HIF design (see, for example, Ref. [24]). Several experiments studying channel formation and beam transport have recently been conducted [65]. After initial experiments at LBNL, an improved experiment was built at the 'Gesellschaft fuer Schwerionenforschung'(GSI). These experiments study the physics of channel formation and its beam transport capabilities, using $\mathrm{GeV}$ ion beams from the linear accelerator UNILAC [65]. The ions have an energy similar to IFE requirements, but the ion current is only in the $\mu \mathrm{A}$ range, in contrast to the several hundred $\mathrm{kA}$ required 


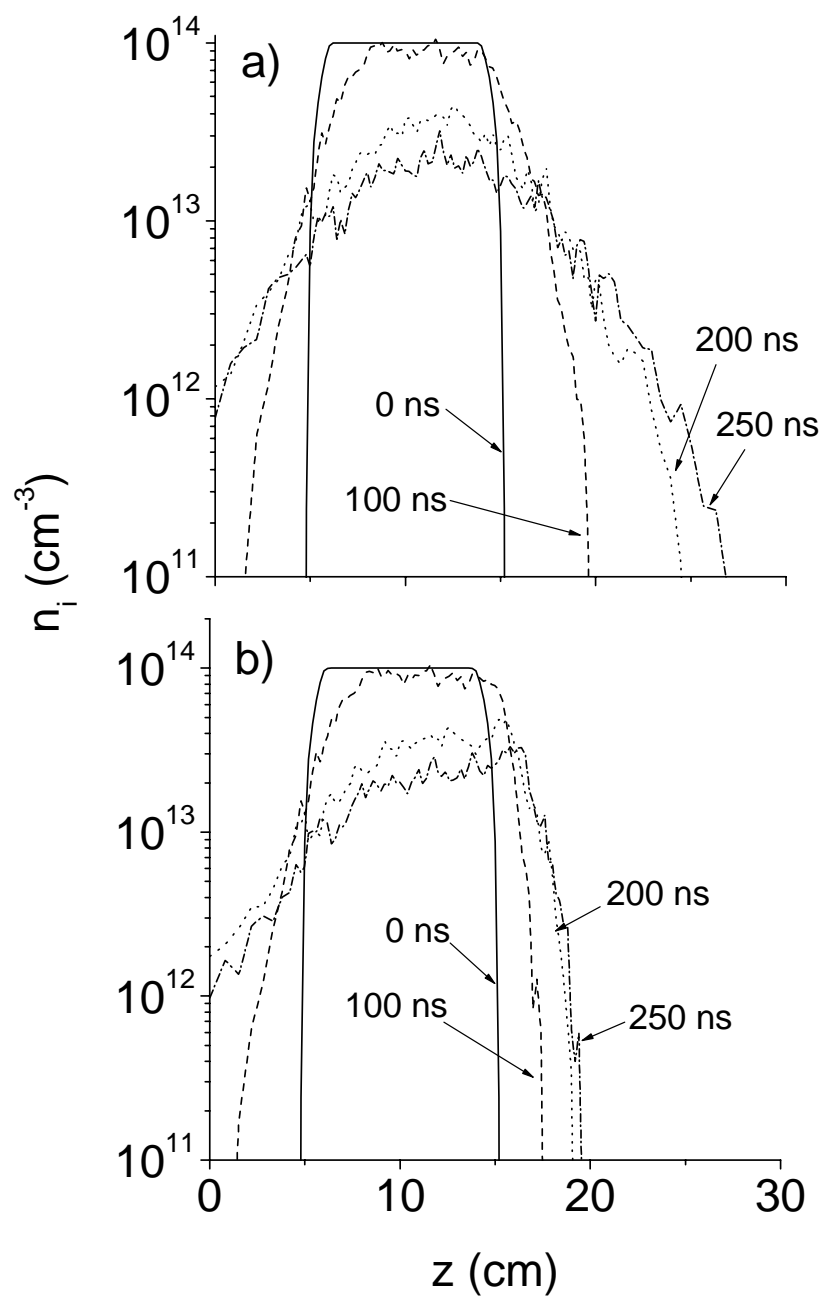

Figure 19: Plasma ion number density as a function of distance along the center $(x=0, y=$ 0 ) of the pipe at different times for a) the case with no applied magnetic field, and b) the case for $B_{y}=0.1 \mathrm{~T}$ for $15 \mathrm{~cm} \leq z \leq 30 \mathrm{~cm}$. 


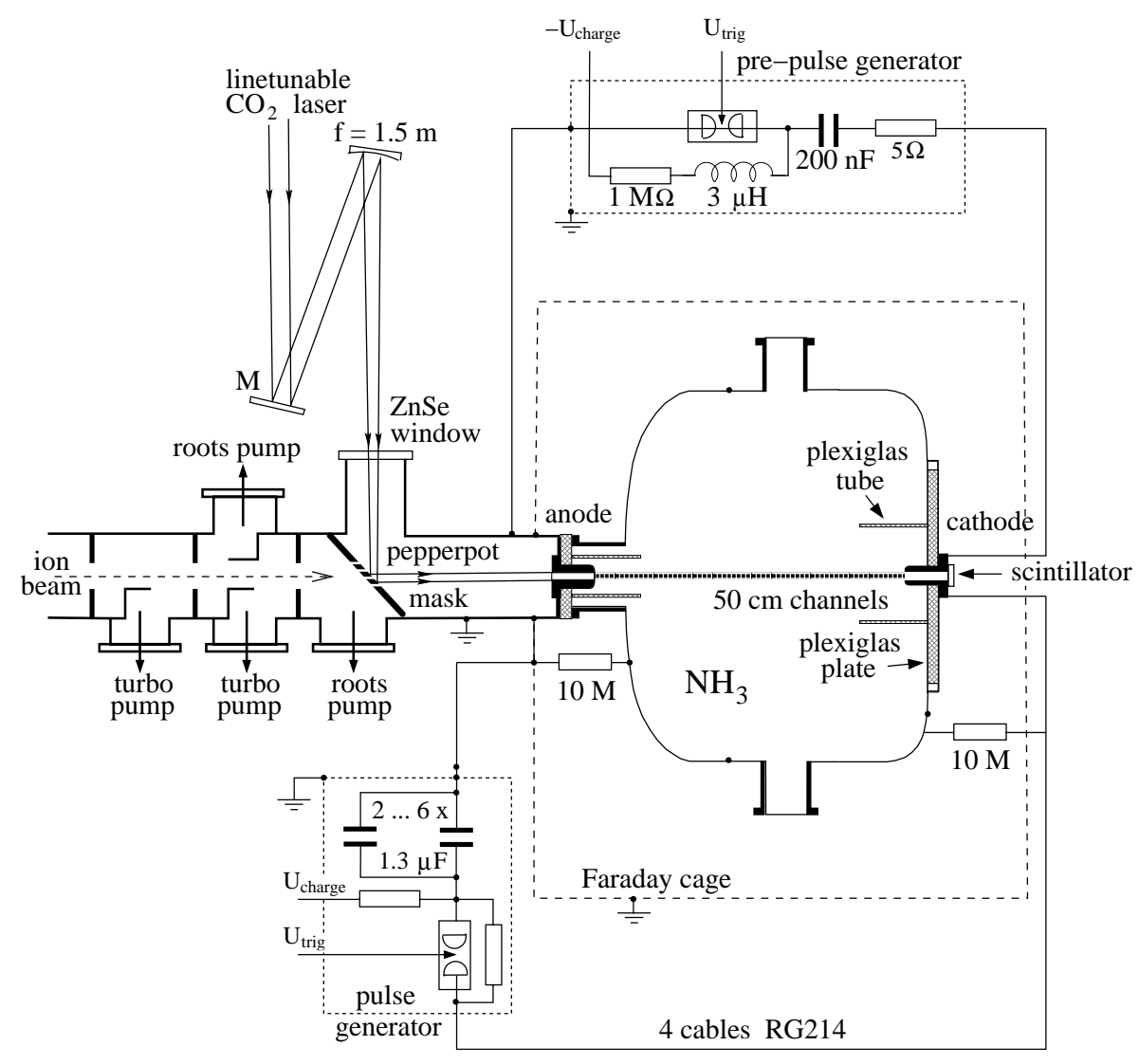

Figure 20: APT experimental setup at GSI.

for a reactor. Therefore, the effects of the beam on the channel dynamics cannot be studied with this experiment, and for now we must rely on numerical simulations.

The setup of the experiment at GSI is shown in Fig. 20 [44]. The central part of the experiment is a metal chamber with a length of $50 \mathrm{~cm}$ and a diameter of $60 \mathrm{~cm}$. The chamber pressure is in the range of 0.5 mbar to 20 mbar. The configuration shown is used to create a straight channel, one anode and one cathode being mounted on the beam axis. A modified setup is used to create crossed channels as required for the IFE concept. In this setup, two anodes are mounted on the beam axis and two cathodes on the perpendicular axis. The chamber has four windows perpendicular to the beam axis, allowing the diagnostic access.

The discharges are created by two pulse generators. A small generator generates a prepulse, which has an energy of $40 \mathrm{~J}$. The main generator is able to generate discharges with energies of up to $3 \mathrm{~kJ}$. 
The chamber gas is heated by a $5-\mathrm{J}, \mathrm{CO}_{2}$ laser; the wavelength can be tuned to the absorption maximum of ammonia, which is used in most of the experiments. In the future, a low energy pulse of the laser PHELIX, which is currently under construction at GSI, might be used instead allowing the efficient heating of other chamber gases such as Xe.

A differential pumping section decreases the gas density to a sufficient vacuum at the connection with the accelerator. Immediately upstream of the chamber a pepperpot mask is mounted allowing measurements of the beam properties. The mask also serves as a mirror for the $\mathrm{CO}_{2}$ laser and it helps to reduce the gas flux upstream towards the accelerator.

\section{B.2 Diagnostics}

A thorough understanding of the channel dynamics is required to make APT a suitable candidate for IFE. It is, therefore, necessary to determine the fundamental plasma parameters, the electron density and temperature. Furthermore, the beam transport capabilities of the channel and its stability have to be checked.

The radial profile of the electron density is determined with a Michelson interferometer. An alternative measurement was obtained by using a spectrometer, yielding agreement with interferometer measurements within the error margins. The spectrometer also yields a profile for the electron temperature.

The stability of the discharge is checked with a digital camera. This further enables investigation of possible breakdown to the chamber walls and estimation of the channel radius.

The channel properties are directly measured with the heavy ion beam. The ions have an energy of about $10 \mathrm{MeV} / \mathrm{u}$, similar to the ion energies considered for IFE. After passing through the chamber, the beam hits a scintillator which is mounted at the end of the cathode. The light emission of the scintillator is measured with a high-speed digital camera (10-ns exposure time), a streak camera, or a framing camera. Comparisons of the measured beam intensity with the results of simple Monte Carlo calculations provides estimates of the radial development of the discharge current density.

A new technique to measure the channel gas density has been developed [66]. The idea is to measure the spreading of an ion beamlet due to collisional scattering with the gas, thereby 


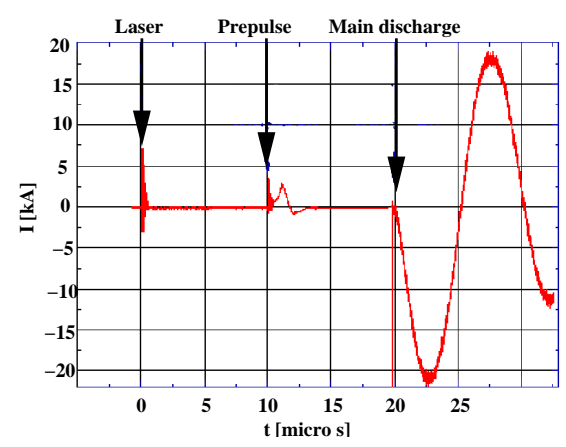

Figure 21: Typical three-stage timing sequence for channel formation.

deducing the average line-integrated gas density. The large fields during the discharges and the ionization of the chamber gas limit the application of the method to the laser heating phase before the prepulse.

\section{B.3 Channel creation}

The creation of the plasma channel is a three stage process, as illustrated in Fig. 21. In the first stage, an infrared laser heats the gas along the axis. The $\mathrm{CO}_{2}$ laser is line-tunable and creates 5 -J pulses with a duration of $1 \mu \mathrm{s}$. The laser absorption is gas dependent. Most experiments are done with ammonia, the laser being tuned to the absorption peak at $950 \mathrm{~cm}^{-1}$. Figure 22 shows the development of the gas density in the first $20 \mu \mathrm{s}$ after heating; the density development is calculated with CYCLOPS, a 1-D axisymmetric MHD code developed at LBNL. The simulation is initialized with a Gaussian temperature profile and a homogeneous gas density; its parameters are determined from measurements with a pyroelectric detector. However, absorption is not uniform along the z-axis; the intensity of the laser follows the exponential law

$$
I(z)=I(0) \cdot \exp [-\alpha z]
$$

with a gas dependent absorption coefficient $\alpha$. Choosing the optimum gas pressure allows maximum absorption and, thus, achieving sufficient uniformity of the temperature profile along the axis. The density profile closely follows the laser footprint while slowly expanding outwards. The calculations for 15 mbar ammonia shows a density reduction by $73 \%$ in the center; the density hole is surrounded by a wall with $34 \%$ increased gas density. The 


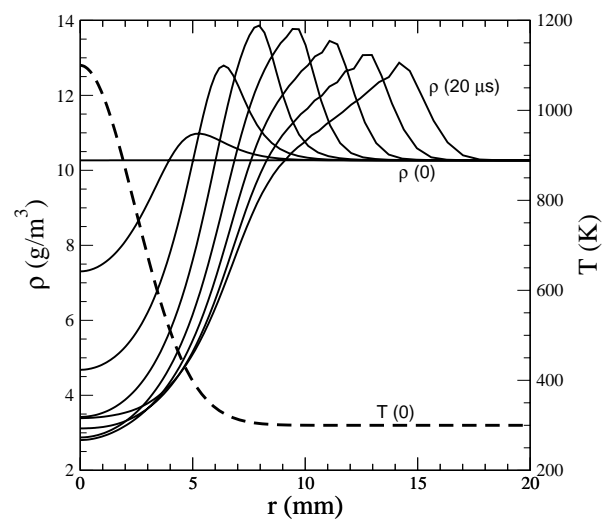

Figure 22: The calculated temperature profile immediately after the laser pulse $T(0)$ (dashed lines) and the density evolution from 0-20 $\mu$ s (solid lines) for 15 mbar ammonia.

rarefaction takes place in the first $10 \mu \mathrm{s}$; afterwards the channel is slowly expanding. In order to benchmark the simulation results, the development of the gas density has also been measured. The results compare favorably with the 1-D code calculations.

By decreasing the pressure, the necessary discharge voltage is lowered. This is a significant effect; a separate measurement was performed to quantify it. For a 15-mbar ammonia atmosphere, the breakdown voltage was reduced by $40 \%$. Thus, the discharge is guided along the channel, preventing a breakdown to the walls.

The density profile also stabilizes the main discharge against kink instabilities. The stabilizing effect of a low density channel surrounded by a homogeneous gas background has been calculated by Mannheimer, et al. [67]. The growth rate of the kink instability is determined by the Alfvén speed

$$
v_{A}=B_{\Theta} \sqrt{\mu_{0} \rho_{c h}}
$$

where $\rho_{c h}$ is the mass density in the channel. The growth rate for a discharge in vacuum is given by $\Gamma_{k i n k}=k \cdot v_{A}, k$ being the wave number of the instability. Taking the gas background into account reduces this by a factor of $\left(\rho_{g} / \rho_{c h}\right)^{1 / 2}$ [67]. Since the channel is surrounded by a wall of increased density, the actual effect is even greater.

When the gas density reaches its minimum, a spark triggers the prepulse. The purpose of the prepulse is to further rarefy and ionize the gas. The main discharge is triggered several microseconds later. It has an energy of up to $3 \mathrm{~kJ}$ and creates the plasma channel. The time 
development of the channel is illustrated in Fig. 23; the figure juxtaposes the development of the channel with and without prepulse. The pictures are composed of photographs of the discharge, made with a high-speed camera with an exposure time of 10 ns. As illustrated in the left-side picture, the discharge in 20 mbar ammonia grows unstable after $3 \mu \mathrm{s}$, leading to the disruption of the channel after $8 \mu \mathrm{s}$. In contrast, the channel created with an additional prepulse remains stable over the observed $9 \mu \mathrm{s}$. The stabilizing effect of the prepulse is less distinctive for lower gas pressures. It was possible to create stable discharges for some low pressure noble gases without a prepulse.

So far, only straight channels were discussed. A critical issue is the creation of crossed channels, which requires the discharge channel to make a turn by $90^{\circ}$. A modified experimental setup was used to create the crossed channels. As illustrated in Fig. 24, it is possible to create discharge channels with a sharp bend by guiding the discharge with a laser.

\section{B.4 Beam transport}

The UNILAC linear accelerator at GSI is used to study the beam transport properties of the plasma channel. The beam is shaped by a pepperpot mask before entering the chamber. The beam pattern is used to measure channel distortions and determine the focusing capabilities of the channel.

The focusing power of the channel is illustrated in Fig. 25. The left-side picture shows the beam after passing through an evacuated chamber. Because of the low beam currents used in the experiments, space-charge effects are negligible and the resulting intensity profile is identical to the initial one. In contrast, the right-side picture shows the beam after being focused by a high-current discharge. All beamlets execute betatron orbits in the discharge
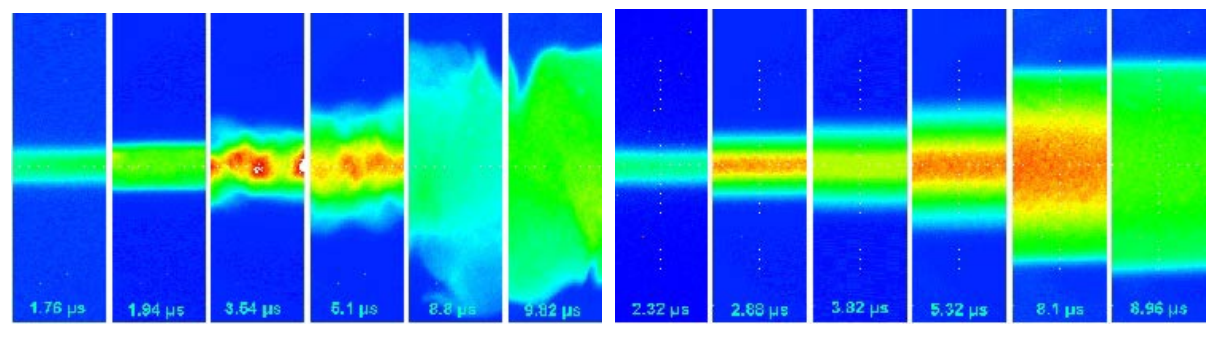

Figure 23: 45-kA discharge in 20 mbar ammonia: without and with 3-kA prepulse. 


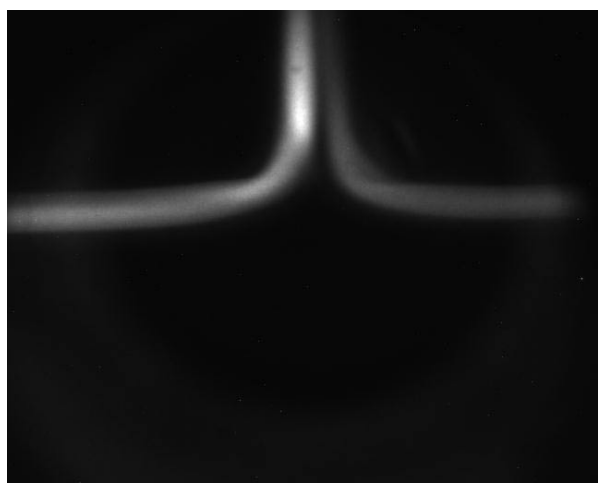

Figure 24: Example illustrating the laser creation of bent discharge channels.
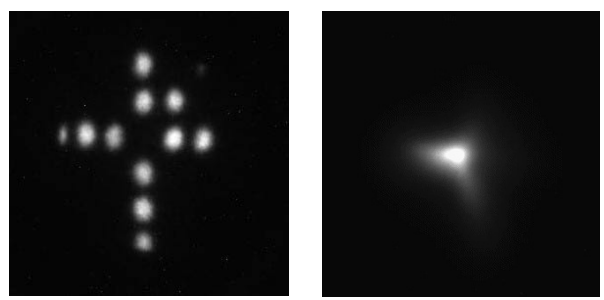

Figure 25: Initial beam profile (left) and focused beam (right). The width of the cross is $1 \mathrm{~cm}$.

channel, and pass through one common focal point. These measurements have been used to infer radial current density profiles of the discharge channel.

\section{Acknowledgments}

Work supported through the ARIES project and by the U.S. DOE through Lawrence Berkeley National Laboratory and the Princeton Plasma Physics Laboratory. C. L. Olson acknowledges discussions with M. Rosenberg, at the University of California, San Diego, on the topics of aerosols and dusty plasmas. The authors thank Sue Welch for technical editing assistance. 


\section{References}

[1] R. O. Bangerter, "The heavy ion fusion program in the USA," Nucl. Instrum. Meth. Phys. Res. A 464, 17 (2001).

[2] A. Friedman, "Overview of heavy ion fusion accelerator research in the U. S.," $A d$ vanced Accelerator Concepts, Tenth Workshop, AIP Conf. Proc. No. 647, edited by C. E. Clayton and P. Muggli (American Institute of Physics, NY), p. 106.

[3] W. R. Meier, B. G. Logan, W. L. Waldron, G.-L. Sabbi, D. A. Callahan, P. F. Peterson, and D. T. Goodin, "Progress toward heavy-ion IFE," Fusion Engineering and Design 63-64, 577 (2002).

[4] M. Tabak and D. Callahan-Miller, "Design of a distributed radiator target for inertial fusion driven from two sides with heavy ion beams," Phys. Plasmas 5, 1895 (1998).

[5] D. A. Callahan-Miller and M. Tabak, "A distributed radiator, heavy ion target driven by Gaussian beams in a multibeam illumination geometry," Nucl. Fusion 39, 883 (1999).

[6] F. Najmabadi, R. Raffray, M. Tillack, D. Goodin, D. Haynes, C. Olson, and the ARIES Team, "Assessment of chamber concepts for inertial fusion energy power plants - the ARIES-IFE study," in the Proc. Intl. Symp. on Inertial Fusion Sci. and Appl., IFSA2001, Kyoto, Japan, Sept. 2001, Elsevier Data Science Library, p. 701.

[7] F. Najmabadi, "Design windows and trade-offs for inertial fusion energy power plants," presented at the Sixth Intl. Conf. on Fusion Nucl. Technol., April 7-12, 2002, San Diego, CA.

[8] A. R. Raffray, D. Haynes, and F. Najmabadi, "IFE chamber walls: requirements, design options, and synergy with MFE plasma facing components," J. Nucl. Materials (2002), in press.

[9] A. R. Raffray, G. Federici, A. Hassanein, and D. Haynes, "Dynamics chamber armor behavior in IFE and MFE," Fusion Eng. Design 63-64, 597 (2002).

[10] D. R. Olander, G. T. Fukuda, and C. F. Baes, Jr., "Equilibrium pressures over $\mathrm{BeF}_{2} / \mathrm{LiF}(\mathrm{FLIBE})$ molten mixtures," Fusion Sci. Technol. 41, 141 (2002).

[11] R. W. Moir, R. L. Bieri, X. M. Chen, T. J. Dolan, et al., "HYLIFE-II: a molten-salt inertial fusion energy power plant design - final report," Fusion Technol. 25, 5 (1994).

[12] R. W. Moir, "The high-yield lithium-injection fusion-energy (HYLIFE)-II inertial fusion energy (IFE) power plant concept and implications for IFE," Phys. Plasmas 2, 2447 (1995).

[13] R. W. Moir, "Chamber, target and final focus integrated design," Nucl. Instrum. Meth. Phys. Res. A 464, 140 (2001).

[14] P. F. Peterson, "Design methods for thick-liquid protection of inertial fusion chambers," Fusion Technol. 39, 702 (2001). 
[15] R. Abbott, S. Pemberton, P. F. Peterson, G.-P. Sun, P. Wright, R. Holmes, J. Latkowski, R. Moir, and K. Springer, "Cylindrical liquid jet grids for beam-port protection of thickliquid heavy-ion fusion target chambers," Fusion Technol. 39, 732 (2001).

[16] R. R. Peterson, D. A. Haynes, Jr., I. E. Golovkin, and G. A. Moses, "Inertial fusion energy target output and chamber response: calculations and experiments," Phys. Plasmas 9, 2287 (2002).

[17] M. S. Tillack, F. Najmabadi, L. A. El-Guebaly, R. R. Peterson, D. T. Goodin, K. R. Schultz, W. R. Meier, J. Perkins, D. A. Petti, J. D. Sethian, and L. M. Waganer, "ARIES inertial fusion chamber assessment," Fusion Tech. 39, 343 (2001).

[18] J. D. Sethian, S. P. Obenschain, M. Myers, A. J. Schmitt, et al., "Fusion energy research with lasers, direct drive targets, and dry wall chambers," submitted to Nucl. Fusion (2002).

[19] N. Sviatoslavky, M. E. Sawan, R. R. Peterson, et al., "A KrF laser driven inertial fusion reactor SOMBRERO", Fusion Technol. 21, 1470 (1992).

[20] G. L. Kulcinski, R. R. Peterson, L. J. Winterberg, E. A. Mogahed, and I. N. Sviatoslavsky, "Dry wall chamber issues for the SOMBRERO laser fusion power plant," Fusion Engineering and Design 60, 3 (2002).

[21] C. L. Olson, "Chamber transport," Nucl. Instrum. Meth. Phys. Res. A 464, 118 (2001).

[22] D. A. Callahan, "Chamber propagation physics for heavy ion fusion," Fusion Engineering and Design 32-33, 441 (1996).

[23] D. R. Welch, D. V. Rose, B. V. Oliver, T. C. Genoni, R. E. Clark, C. L. Olson, and S. $\mathrm{S}$. Yu, "Simulations of intense heavy ion beams propagating through a gaseous fusion target chamber," Phys. Plasmas 9, 2344 (2002).

[24] S. Yu, S. Eylon, T. Fessenden, E. Henestroza, et al., "Plasma-channel-based reactor and final transport," Nucl. Instrum. Meth. Phys. Res. A 415, 174 (1998).

[25] W. R. Meier, D. A. Callahan-Miller, J. F. Latkowski, B. G. Logan, J. D. Lindl, and P. F. Peterson, "An engineering test facility for heavy ion fusion — options and scaling," Fusion Technol. 39, 671 (2001)

[26] W. R. Meier, "Overview of chamber and target technology R\&D for heavy ion fusion," Nucl. Instrum. Meth. Phys. A 464, 103 (2001).

[27] W. R. Meier, "An integrated research plan for the IFE element of the Virtual Laboratory for Technology," Fusion Eng. Design 60, 37 (2002).

[28] W. R. Meier, J. J. Barnard, and R. O. Bangerter, "A 3.3 MJ, Rb ${ }^{+1}$ driver design based on an integrated systems analysis," Nucl. Instrum. Meth. Phys. A 464, 433 (2001). 
[29] S. S. Yu, W. R. Meier, R. P. Abbott, J. J. Barnard, T. Brown, D. A. Callahan, P. Heitzenroeder, J. F. Latkowski, B. G. Logan, S. J. Pemberton, P. F. Peterson, D. V. Rose, G-L. Sabbi, W. M. Sharp, and D. R. Welch, "A robust point design for heavy ion fusion," submitted to Fusion Sci. Technol. (2002).

[30] D. A. Callahan, M. C. Hermann, and M. Tabak, "Progress in heavy ion target capsule and hohlraum design," to appear in Lasers and Particle Beams (2002).

[31] C. L. Olson, "HIF transport issues for $p>10^{-3}$ torr and $Z>1$," in Heavy Ion Inertial Fusion, edited by M. Reiser, T. Godlove, and R. Bangerter (AIP Conf. Proc. 152, Am. Inst. Phys., NY, 1986), p. 215.

[32] LSP is a software product of Mission Research Corporation (http://www.mrcabq.com).

[33] D. R. Welch, D. V. Rose, W. M. Sharp, and S. S. Yu, "Effects of pre-neutralization on heavy-ion fusion chamber transport," to appear in Lasers and Particle Beams (2002).

[34] C. L. Olson, "Ion beam propagation and focusing," J. Fusion Energy 1, 309 (1982).

[35] D. R. Welch, D. V. Rose, B. V. Oliver, and R. E. Clark, "Simulation techniques for heavy ion fusion chamber transport," Nucl. Instrum. Meth. Phys. Res. A 464, 134 (2001).

[36] D. V. Rose, D. R. Welch, B. V. Oliver, R. E. Clark, W. M. Sharp, A. Friedman, "Ballistic-neutralized chamber transport of intense heavy ion beams," Nucl. Instrum. Meth. Phys. Res. A 464, 299 (2001).

[37] W. M. Sharp, D. A. Callahan, M. Tabak, S. S. Yu, P. F. Peterson, D. R. Welch, D. V. Rose, and C. L. Olson, "Modeling chamber transport for heavy-ion fusion" to appear in Fusion Sci. and Technol. (2002).

[38] A. B. Langdon, "Reactor chamber propagation of heavy ion beams," Part. Accel. 37-38, 175 (1992).

[39] W. M. Sharp, D. A. Callahan-Miller, A. B. Langdon, M. S. Armel, and J.-L. Vay, "Improved modeling of chamber transport for heavy-ion fusion," Nucl. Instrum. Meth. Phys. Res. A 464, 284 (2001).

[40] R.C. Davidson, I. D. Kaganovich, W. W. Lee, H. Qin, E. A. Startsev, S. Tzenov, A. Friedman, J. J. Barnard, R. H. Cohen, D. P. Grote, S. M. Lund, W. M. Sharp, C. M. Celata, M. de Hoon, E. Henestroza, E. P. Lee, S. S. Yu, J.-L. Vay, D. R. Welch, D. V. Rose, and C. L. Olson, "Overview of theory and modeling in the Heavy Ion Fusion Virtual National Laboratory," to appear in Lasers and Particle Beams (2002).

[41] D. R. Welch, T. C. Genoni, D. V. Rose, B. V. Oliver, R. E. Clark, C. L. Olson, and S. $\mathrm{S}$. Yu, "Assisted pinched transport of heavy ion beams in a fusion chamber," submitted to Phys. Plasmas (2002).

[42] M. C. Vella, T. J. Fessenden, W. Leemans, S. Yu, and A. Tauschwitz, "Plasma pinch for final focus and transport," Nucl. Instrum. Meth. Phys. Res. A 415, 193 (1998). 
[43] D. M. Ponce, C. Niemann, S. S. Yu, W. P. Leemans, T. J. Fessenden, G. Dahlbacka, W. M. Sharp, and A. Tauschwitz, "Diagnostics of plasma channel for HIF transport," Nucl. Instrum. Meth. Phys. Res. A 464, 331 (2001).

[44] C. Niemann, A. Tauschwitz, D. Penache, S. Neff, R. Knobloch, R. Birkner, R. Presura, D. H. H. Hoffmann, S. S. Yu, and W. M. Sharp, "Initialization of long, free-standing $z$ discharges by $\mathrm{CO}_{2}$ laser gas heating," J. Appl. Phys. 91, 617 (2002).

[45] J. N. Olsen and R. J. Leeper, "Ion beam transport in laser-initiated discharge channels," J. Appl. Phys. 53, 3397 (1982).

[46] T. Ozaki, S. Miyamota, K. Imasaki, and C. Yamanaka, "Light ion beam transport in plasma channels," J. Appl. Phys. 58, 2145 (1985).

[47] J. M. Neri, P. F. Ottinger, D. V. Rose, P. J. Goodrich, D. D. Hinshelwood, D. Mosher, S. J. Stephanakis, and F. C. Young, "Intense ion-beam-transport experiments using a z-discharge plasma channel," Phys. Fluids B 5, 176 (1993).

[48] E. Boggash, A. Tauschwitz, H. Wahl, K.-G. Dietrich, D. H. H. Hoffmann, W. Laux, M. Stetter, and R. Tkotz, "Plasma lens fine focusing of heavy ion beams," Appl. Phys. Lett. 60, 2475 (1992).

[49] R. R. Peterson and C. L. Olson, "Pre-formed plasma channels for ion beam fusion," Laser Interaction and Related Plasma Phenomena (AIP Conf. Proc. 406, Am. Inst. Phys., Woodbury, NY, 1997), p. 259.

[50] R. R. Peterson, M. E. Sawan, S. Yu, and R. Moir, "Target chamber considerations for heavy ion transport in plasma channels," Nucl. Instrum. Meth. Phys. Res. A 415, 182 (1998).

[51] D. R. Welch, C. L. Olson, and T. W. L. Sanford, "Simulation of charge-particle beam transport in a gas using a hybrid particle-fluid plasma model," Phys. Plasmas 1, 764 (1994).

[52] D. R. Welch and C. L. Olson, "Self-pinched transport for ion-driven inertial confinement fusion," Fusion Eng. Design 32-33, 477 (1996).

[53] P. F. Ottinger, F. C. Young, S. J. Stephanakis, D. V. Rose, J. M. Neri, B. V. Weber, M. C. Myers, D. D. Hinshelwood, D. Misher, C. L. Olson, and D. R. Welch, "Self-pinched transport of an intense proton beam," Phys. Plasmas 7, 346 (2000).

[54] M. Gryzinski, "Two-particle collisions. II. Coulomb collisions in the laboratory system of coordinates," Phys. Rev A 138, A322 (1965).

[55] D. V. Rose, T. C. Genoni, and D. R. Welch, "Steady-state erosion of propagating ion beams" Phys. Plasmas 9, 1053 (2002).

[56] J. D. Jackson, Classical Electrodynamics (John Wiley, NY, 1975), p. 618.

[57] H. A. Bethe, "Moleire's theory of multiple scattering," Phys. Rev. 89, 1256 (1953). 
[58] H. E. Hesketh, Fine Particles in Gaseous Media (Lewis Pub., 1986), pp. 10, 74.

[59] E. C. Whipple, "Potentials of surfaces in space," Rep. Prog. Phys. 44, 1197 (1981).

[60] V. W. Chow, D. A. Mendis, M. Rosenburg, "Role of grain size and particle velocity distribution in secondary electron emission in space plasmas," J. Geophys. Res. 98, 19065 (1993).

[61] M. Rosenberg, "Ion-dust streaming instability in processing plasmas," J. Vac. Soc. Technol. A 14, 631 (1996).

[62] C.S. Debonnel, G.T. Fukuda, P.M. Bardet, P.F. Peterson, Control of the Heavy-Ion Beam Line Gas Pressure and Density in the HYLIFE Thick-Liquid Chamber, Fusion Eng. Design 63-64, 647 (2002).

[63] C. S. Debonnel, D. R. Welch, D. V. Rose, S. S. Yu, and P. F. Peterson, "Gas transport and density control in the HYLIFE heavy-ion beam lines," to appear in Fusion Sci. and Technol. (2002).

[64] W. Peter, A. Ron, and N. Rostoker, "Instability of the boundary layer between a streaming plasma and a vacuum magnetic field," Phys. Fluids 26, 2276 (1983).

[65] A. Tauschwitz, C. Niemann, D. Penache, R. Birkner, D. H. H. Hoffmann, R. Kobloch, S. Neff, R. Presura, D. Ponce, F. Rosmoj, and S. Yu, "Plasma channel transport for heavy ion fusion: investigation of beam transport, channel initiation and stability," in the Proceedings of the 14th Intl. Conf. on High-Power Particle Beams, BEAMS 2002, American Institite of Physics Conf. Proceedings 650, edited by T. A. Mehlhorn and M. A. Sweeney (American Institute of Physics, Melville, NY, 2002), p. 391.

[66] S. Neff, A. Tauschwitz, C. Niemann, D. Penache, D. H. H. Hoffmann, S. S. Yu, and W. M. Sharp, "Gas density measurements with heavy ion beams," to appear in J. Appl. Phys. (2003).

[67] W. M. Manheimer, M. Lampe, and J. P. Boris, "Effect of a surrounding gas on magnetohydrodynamic instabilities in Z pinch," Phys. Fluids 16, 1126 (1973). 